OPEN ACCESS

Edited by:

Guillermo Garcia-Cardena,

Harvard Medical School,

United States

Reviewed by:

Daniel Carneiro Moreira,

University of Brasilia, Brazi

Elena Grasselli,

University of Genoa, Italy

*Correspondence:

Yunfang Gao

gaoyunf@nwu.edu.cn

tThese authors have contributed equally to this work

Specialty section: This article was submitted to

Oxidant Physiology,

a section of the journal

Frontiers in Physiology

Received: 30 July 2019 Accepted: 08 November 2019 Published: 22 November 2019

Citation:

Wei Y, Zhang J, Yan X, Peng $X$, $X u S$, Chang $H$, Wang $H$ and Gao $Y$

(2019) Remarkable Protective Effects of Nrf2-Mediated Antioxidant

Enzymes and Tissue Specificity in Different Skeletal Muscles of Daurian Ground Squirrels Over the Torpor-Arousal Cycle.

Front. Physiol. 10:1449.

doi: 10.3389/fphys.2019.01449

\section{Remarkable Protective Effects of Nrf2-Mediated Antioxidant Enzymes and Tissue Specificity in Different Skeletal Muscles of Daurian Ground Squirrels Over the Torpor-Arousal Cycle}

\author{
Yanhong Wei ${ }^{1,2+}$, Jie Zhang ${ }^{1 \dagger}$, Xia Yan ${ }^{1}$, Xin Peng ${ }^{1}$, Shenhui Xu ${ }^{1}$, Hui Chang ${ }^{1}$, \\ Huiping Wang ${ }^{1}$ and Yunfang Gao ${ }^{1 *}$
}

'Key Laboratory of Resource Biology and Biotechnology in Western China, Ministry of Education, College of Life Sciences, Northwest University, Xi'an, China, ${ }^{2}$ School of Basic Medical Sciences, Ningxia Medical University, Yinchuan, China

Hibernating mammals experience conditions of extreme oxidative stress, such as fasting, muscle disuse, and repeated hypoxic ischemia-reperfusion, during the torporarousal cycle. Despite this, they experience little oxidative injury and are thus an interesting model of anti-oxidative damage. Thus, in the current study, we explored the levels and underlying mechanism of oxidative stress and antioxidant capacity in three skeletal muscles [slow-twitch soleus (SOL), fast-twitch extensor digitorum longus (EDL), and mixed gastrocnemius (GAS)] of Daurian ground squirrels (Spermophilus dauricus) during hibernation. Results showed that hydrogen peroxide content in the EDL and GAS decreased significantly during pre-hibernation (PRE) and late torpor $(L T)$ compared to levels in the summer active (SA) group. Furthermore, relative to SA levels, malondialdehyde content decreased significantly during interbout arousal (IBA) and early torpor (ET) in all three skeletal muscles and decreased in the EDL and GAS during LT. Compared with the SA group, glutathione peroxidase 1 (GPX1) and catalase (CAT) protein expression in the SOL and superoxide dismutase 1 (SOD1) and SOD2 expression in the GAS increased significantly during the entire hibernation season. Furthermore, SOD1 in the IBA group and CAT and GPX1 in the ET and LT groups increased significantly in the EDL. The activities of most tested antioxidant enzymes were higher in the IBA group than in the LT group, whereas CAT remained highly active throughout the hibernation season in all three muscles. Nrf2 and p-Nrf2 protein levels were significantly elevated in the SOL and EDL during hibernation, and increased during the PRE, IBA, and ET states in the GAS. Thus, activation of the Nrf2/Keap1 antioxidant pathway resulted in the elimination of excess reactive oxygen species (ROS). Specifically, ROS levels were maintained at physiological levels by the up-regulation of antioxidant enzyme expression in skeletal muscles under oxidative stress during hibernation, thus preventing oxidative injury over the torpor-arousal cycle. Different antioxidant patterns and oxidative stress levels were also observed among the different skeletal muscles of hibernating Daurian ground squirrels.

Keywords: hibernation, oxidative stress, antioxidant enzymes, Nrf2/Keap1 signaling pathway, skeletal muscle 


\section{INTRODUCTION}

Hibernating animals display specific adaptations and physiological functions suitable for the conditions experienced during the torpor-arousal period, i.e., low temperature, fasting, immobilization, and repeated hypoxic ischemiareperfusion. Torpor involves the coordinated suppression of many physiological functions, including metabolic rate (to $1-5 \%)$, body temperature $(\mathrm{Tb})\left(1-5^{\circ} \mathrm{C}\right)$, heart rate, blood flow, and respiration (Geiser, 2004; Storey, 2010). However, intermittent episodes of arousal (generally $<24 \mathrm{~h}$ ) and euthermia are also experienced during torpor, involving the rapid reperfusion of oxygen and blood to all tissues (Carey et al., 2003; Storey, 2010). For example, oxygen consumption in the liver mitochondria of thirteen-lined ground squirrels (Spermophilus tridecemlineatus) increases dramatically during arousal (by 36-fold) compared with that during torpor (Muleme et al., 2006) and oxygen consumption in Arctic ground squirrels (S. parryii) increases significantly at different points during arousal in hibernation (Toien et al., 2001). The dramatic changes in physiological functions experienced by hibernators during the torpor-arousal cycle are similar to repeated ischemia-reperfusion (Ma et al., 2005; Ni and Storey, 2010). For non-hibernating animals (including humans), reduced blood perfusion can cause abnormal tissue and cellular metabolism, resulting in ischemic injury; furthermore, when blood reperfusion is restored, tissue and cellular damage can worsen as excessive free radicals can attack tissues and cells after they regain blood supply (Wang and Yin, 2013). As important hindlimb tissues, skeletal muscles perform many vital motor functions and are highly sensitive to ischemia (Blaisdell, 2002). Ischemia-reperfusion, with the massive production of reactive oxygen species (ROS), can damage the vascular endothelium system and increase the permeability of blood vessel walls. This can, in turn, lead to skeletal muscle edema, platelet adhesion and aggregation in microvessels, and deeper muscle color, and in severe cases, fibrosis, contracture, and necrosis of limbs as well as life-threatening failure of distant internal organs (such as the heart and kidney) (Carden and Granger, 2000; Kalyanaraman, 2013). Therefore, oxidative stress in skeletal muscles is a crucial area of research. Previous studies on non-hibernating animals have shown that an increase in ROS to the point where antioxidant scavenging capacity is exceeded is a significant cause of ischemia-reperfusion injury in skeletal muscles (Liu et al., 2007; Li and Zhang, 2015). In contrast, despite experiencing high oxidative stress during hibernation, including repeated ischemia-reperfusion, hibernating mammals exhibit no (or limited) evidence of oxidative injury, cellular stress, inflammation, or neuronal pathology (Ma et al., 2005). Therefore, understanding the antioxidant defense ability and regulation mechanism of skeletal muscles in hibernating animals is an important issue.

Reactive oxygen species are intermediate products of cellular metabolism and play important roles in maintaining intracellular homeostasis and signal transduction (Espinosa-Diez et al., 2015). Under conditions of excessive ROS, organismal endogenous antioxidant defenses are initiated to maintain redox homeostasis; however, if the accumulation of ROS exceeds the body's antioxidant elimination capacity, oxidative damage can occur (Espinosa-Diez et al., 2015). Previous studies have found that malondialdehyde (MDA), a biomarker of lipid peroxidation, significantly increases in the skeletal muscles of non-hibernating rats (Lin et al., 2018) and in the serum and hindlimb skeletal muscles of non-hibernating rabbits following ischemiareperfusion (Li et al., 2017; Zhang et al., 2018). Furthermore, the activities of multiple antioxidant enzymes, including superoxide dismutase (SOD), catalase (CAT), and glutathione peroxidase (GPx), are significantly reduced in the skeletal muscles of nonhibernating rabbits experiencing ischemia-reperfusion (Zhang et al., 2018), with changes also observed in apoptosis gene expression in skeletal muscle fibers (e.g., increased Bax and decreased Bcl-2 expression) (Li et al., 2017; Zong et al., 2017). The modulation of antioxidants during hibernation has been investigated extensively. Prior studies have found that the maximum rate of ROS production by mitochondria isolated from the skeletal muscles of hibernating thirteen-lined ground squirrels does not differ between torpor and interbout arousal (Brown et al., 2012). Furthermore, the expression of multiple antioxidant proteins, including SOD1, SOD2, CAT, and GPx, increases significantly in the skeletal muscles of European ground squirrels (S. citellus) (Vucetic et al., 2013) and thirteen-lined ground squirrels during torpor (Allan and Storey, 2012; Xu et al., 2013; Wu and Storey, 2014). Although such studies have explored the antioxidant defenses of skeletal muscles in hibernating animals, they have not distinguished the susceptibility of different types of skeletal muscles to oxidative stress. Based on proteomic analysis, our laboratory previously showed that the expression of SOD1 and CAT increases in the soleus (SOL) of Daurian ground squirrels (S. dauricus) in post-hibernation, indicating that the SOL exhibits a stress reaction and increased sensitivity to oxidants (Chang et al., 2016). In conclusion, existing research suggests that mammalian hibernators establish efficient antioxidant defense measures to cope with the challenge of oxidative stress during torpor-arousal cycles. Although skeletal muscle is an essential hindlimb tissue in animal movement, few studies have examined oxidative stress and antioxidant defense in the different types of skeletal muscles of hibernators, which is important for understanding the mechanisms of skeletal muscle weight and functional maintenance during hibernation.

Antioxidant defenses are regulated by many transcription factors, with the NF-E2-related factor 2 (Nrf2)/Kelch-like ECHassociated protein 1 (Keap1) signaling pathway recognized as one of the most important for antioxidant damage (Espinosa-Diez et al., 2015). Under normal circumstances, the Nrf2 protein and its inhibitor (Keap1) undergo cytoplasmic interaction and proteasomic degradation, which suppresses Nrf2 transcriptional activity (Sun et al., 2007). Under increased ROS production, however, proteasomic degradation is hampered. This initiates the release of Nrf2 from cytoplasmic Keap1/Nrf2 complexes, followed by nuclear translocation and accumulation as well as heterodimeric reactions with cis-regulatory elements located on the promoter regions of genes that encode defense proteins/enzymes (Bryan et al., 2013; Lushchak, 2014). Up-regulated antioxidant enzymes include SOD (Park and Rho, 2002), CAT, peroxidases (Lushchak, 2011), thioredoxin 
(Kim et al., 2001), glutathione-S-transferases (GST) (Chanas et al., 2002), and glutamate-cysteine ligase (GCL) (Lushchak, 2012). Previous studies have shown that Nrf2 protein expression is significantly higher in the gastrocnemius (GAS) muscle of rats under ischemia-reperfusion than under normal conditions, indicating that the body can resist ROS through the Nrf2/Keap1 pathway (Zong et al., 2017). As mentioned earlier, hibernators up-regulate the expression of antioxidant enzymes in skeletal muscles during hibernation (Allan and Storey, 2012; Vucetic et al., 2013); however, the mechanism of this up-regulation and whether it is related to the Nrf2/Keap1 antioxidant defense pathway remain unknown.

Skeletal muscles are also essential during interbout arousal due to their role in shivering thermogenesis, which helps the body return to euthermia (Dark and Miller, 1997). Theoretically, however, this activity may generate excessive ROS production due to the swift recovery of oxygen consumption and mitochondrial respiration during arousal (Ruuge et al., 1991; Hermes-Lima and Zenteno-Savin, 2002; Sanderson et al., 2013). Moreover, different types of muscle fibers exhibit differences in mitochondrial productivity, energy metabolism, and effects on the body. Therefore, it is important to understand the levels and underlying mechanism of oxidative stress and antioxidant capacity in different skeletal muscles of mammalian hibernators, particularly the effects of ischemia-reperfusion and antioxidant defense strategies during the torpor-arousal cycle. Here, we investigated how the antioxidant system in the skeletal muscles of Daurian ground squirrels responds to conditions experienced during the torpor-arousal cycle (e.g., low temperature, fasting, mechanical unloading, and repeated hypoxic ischemia-reperfusion). The SOL muscle consists of slow-twitch oxidative type fibers (i.e., myosin heavy-chain type I, MHC I) (Fu et al., 2016; Wei et al., 2018a), which contain a large number of mitochondria and rely on aerobic oxidation to generate energy. Conversely, the extensor digitorum longus (EDL) muscle consists of fast-twitch glycolytic type fibers (i.e., MHC II) (Fu et al., 2016; Wei et al., 2018a), which contain fewer mitochondria and rely on anaerobic glycolysis to provide energy. The mixed gastrocnemius (GAS) muscle consists of both oxidative and glycolytic type muscle fibers, and thus both aerobic and anaerobic functions (Fu et al., 2016; Wei et al., 2018a). Therefore, we examined the levels of ROS $\left(\mathrm{H}_{2} \mathrm{O}_{2}\right.$ and $\left.\mathrm{MDA}\right)$, protein expression and activity of multiple antioxidant enzymes (SODs, CAT, and GPx), and expression of the Nrf2/Keap1 signaling pathway in three different skeletal muscles (SOL, EDL, and GAS) of Daurian ground squirrels during different periods of the torpor-arousal cycle.

\section{MATERIALS AND METHODS}

\section{Animals and Groups}

Adult Daurian ground squirrels were caught from the wild. Upon return to the laboratory, animals were maintained under standard conditions at $18-25^{\circ} \mathrm{C}$ and provided with food and water ad libitum until the end of October, as detailed in previous studies (Gao et al., 2012; Yang et al., 2014). Squirrel $\mathrm{Tb}$ was measured via thermal visual imaging (Fluke VT04
Visual IR Thermometer, Fluke, United States). All experiments and protocols were approved by the Laboratory Animal Care Committee of the China Ministry of Health.

Non-reproductive adult squirrels were weight-matched and randomly allocated into one of six groups ( $n=8$ in each group), following established experimental categories, i.e., summer active, pre-hibernation, interbout arousal, early torpor, late torpor, and post-hibernation groups (Wei et al., 2018b; Zhang et al., 2019). Details on the different states are listed in Supplementary Table $\mathbf{S}$ and Figure 1.

\section{Muscle Collection}

For muscle collection, all animals were anesthetized with sodium pentobarbital at a dose of $90 \mathrm{mg} / \mathrm{kg}$. Samples of the three hindlimb skeletal muscles (e.g., slow-twitch SOL, fast-twitch EDL, and mixed GAS) (Figure 2) were immediately removed, dissected, and weighed for determination of muscle wet weight, then subsequently frozen in liquid nitrogen and stored at $-80^{\circ} \mathrm{C}$ until use. Upon completion of surgical intervention, all squirrels were euthanized with sodium pentobarbital via overdose injection.

\section{Quantification of $\mathrm{H}_{2} \mathrm{O}_{2}$ and MDA}

As ROS are short-lived and highly reactive, their exact measurement in tissue samples remains difficult (Kohen and Nyska, 2002; Winterbourn, 2008; Kalyanaraman et al., 2012; Cheng et al., 2018). Here, the measurement of $\mathrm{H}_{2} \mathrm{O}_{2}$ (a significant

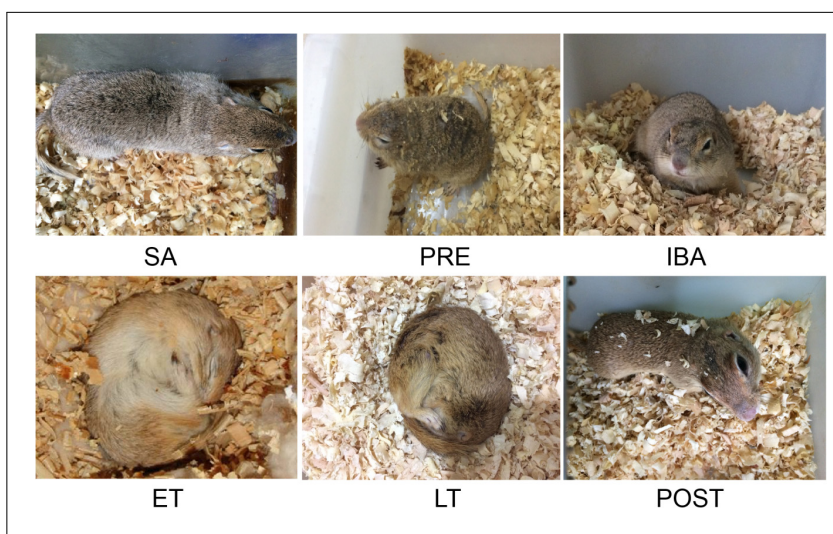

FIGURE 1 | Images of Daurian ground squirrels during different hibernation periods. SA, summer active; PRE, pre-hibernation; ET, early torpor; LT, late torpor; IBA, interbout arousals; POST, post-hibernation.

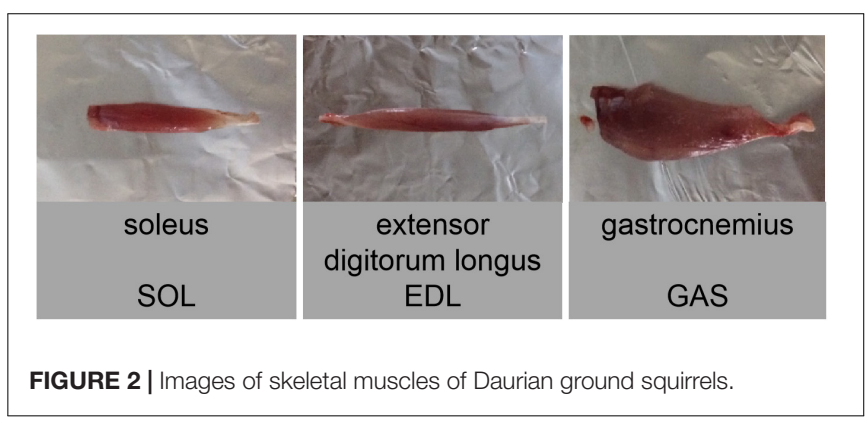


ROS) and MDA (a secondary product) was used as an indicator for the levels of ROS.

Using a high-throughput tissue grinder (Scientz-48, Scientz Biotechnology, Zhejiang, China), frozen SOL, EDL, and GAS samples $(\sim 0.1 \mathrm{~g})$ were homogenized at $4^{\circ} \mathrm{C}$ in phosphatebuffered saline (PBS, $0.9 \mathrm{~mL}$; containing $137 \mathrm{mM} \mathrm{NaCl}$, $2.7 \mathrm{mM} \mathrm{KCl}, 10 \mathrm{mM} \mathrm{Na} 2 \mathrm{HPO}_{4}$, and $2 \mathrm{mM} \mathrm{KH}_{2} \mathrm{PO}_{4}$ ). The tissue homogenates then underwent centrifugation $\left(4^{\circ} \mathrm{C}\right.$, $15 \mathrm{~min}, 3000 \mathrm{rpm}$ ), with the protein concentration in the resulting supernatants determined using a Pierce $^{\mathrm{TM}}$ BCA protein quantitation kit (Thermo Fisher Scientific, Rockford, IL, United States) as per the manufacturer-provided instructions. The remaining supernatants were collected and kept on ice for further use in the following assays.

The concentrations of $\mathrm{H}_{2} \mathrm{O}_{2}$ and $\mathrm{MDA}$ in muscle samples were measured following Wei et al. (2018b) using $\mathrm{H}_{2} \mathrm{O}_{2}$ and MDA assay kits (Nanjing Jiancheng Bioengineering Institute, China), respectively, in accordance with the manufacturer's protocols. The peroxo molybdate acid compound can act as a quantitative $\mathrm{H}_{2} \mathrm{O}_{2}$ indicator. Specifically, $\mathrm{H}_{2} \mathrm{O}_{2}$ can react with molybdic acid to form a stable peroxo molybdic acid compound, which exhibits maximum absorption at $405 \mathrm{~nm}$. Therefore, the content of the compound can be measured at $405 \mathrm{~nm}$ via spectrophotometry (Shimadzu UV-2550, Kyoto, Japan). Muscle $\mathrm{H}_{2} \mathrm{O}_{2}$ content was then determined by comparing its $\mathrm{OD}_{405}$ value against those of the $\mathrm{H}_{2} \mathrm{O}_{2}$ standards.

As an index of oxidative damage, and the level of MDA can be used to indicate the level oxidative stress. Specifically, MDA readily reacts with thiobarbituric acid (TBA) to generate an MDA-TBA adduct (a type of thiobarbituric acid reactive substance, TBARS), which can be quantified colorimetrically. Here, the clarified supernatant derived from the skeletal muscle homogenate was mixed with the assay reagent containing TBA and butylated hydroxytoluene (BHT), with the latter used to reduce any artifactually formed lipid peroxides. The mixture was heated at $100^{\circ} \mathrm{C}$ for $40 \mathrm{~min}$. After cooling, the mixture was centrifuged at $3000 \mathrm{rpm}$ for $15 \mathrm{~min}$ at $4^{\circ} \mathrm{C}$. The absorbance of the supernatants was then measured at $532 \mathrm{~nm}$ via spectrophotometry (Shimadzu UV-2550, Kyoto, Japan). Muscle MDA concentration was then determined by comparing its $\mathrm{OD}_{532}$ value against those of the MDA standards.

\section{Antioxidant Activity Assay}

For the determination of antioxidant enzyme activity, frozen skeletal muscle tissues $(\sim 0.1 \mathrm{~g})$ were homogenized in icePBS $(0.9 \mathrm{~mL}$; containing $137 \mathrm{mM} \mathrm{NaCl}, 2.7 \mathrm{mM} \mathrm{KCl}$,

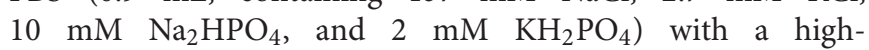
throughput tissue grinder (Scientz-48, Scientz Biotechnology, Zhejiang, China). The tissue homogenates then underwent centrifugation $\left(4^{\circ} \mathrm{C}, 15 \mathrm{~min}, 3000 \mathrm{rpm}\right)$, with the protein concentration in the resulting supernatants determined using a Pierce ${ }^{\mathrm{TM}}$ BCA protein quantitation kit (Thermo Fisher Scientific, Rockford, IL, United States) as per the manufacturerprovided instructions. The remaining supernatants were kept on ice and used for the measurement of SOD, GPx, CAT, and TAC levels. In accordance with previous research (Wei et al., 2018b), antioxidant activity and total antioxidant capacity
(TAC) in the muscle homogenates were ascertained using specific SOD, GPx, CAT, and TAC assay kits (Nanjing Jiancheng Bioengineering Institute, China) as per the manufacturer's respective instructions, with experimental procedures and principles briefly described below. All antioxidant enzyme activities were measured at room temperature.

One unit of total SOD activity corresponded to a SOD inhibition rate of $50 \%$ per milligram of tissue protein in $1 \mathrm{ml}$ of reaction solution (U/mgprot). One unit of CAT enzyme activity was defined as the amount of enzyme capable of directly decomposing $\mathrm{H}_{2} \mathrm{O}_{2}$, in a rate of $1 \mu \mathrm{mol} / \mathrm{min}$, and one unit of GPx enzyme activity was defined as the amount of enzyme capable of directly consume substrate (GSH) in a rate of $1 \mu \mathrm{mol} / \mathrm{min}$.

Superoxide dismutase activity was determined based on the auto-oxidation of hydroxylamine, followed by colorimetric (developed purple color) quantification at $550 \mathrm{~nm}$. Briefly, the superoxide anion $\left(\mathrm{O}_{2}{ }^{-} \cdot\right)$ is produced by the xanthine and xanthine oxidase reaction, and $\mathrm{O}_{2}{ }^{-}$-oxidized hydroxylamine forms nitrite under the action of the color developer. When the sample to be tested contains SOD, it has a specific inhibitory effect on $\mathrm{O}_{2}{ }^{-}$, which reduces the formation of nitrite. Under the colorimetric test, the absorbance value of the tube is lower than the absorbance value of the control tube, and calculation can be performed.

GPx can catalyze the reaction of $\mathrm{H}_{2} \mathrm{O}_{2}$ with reduced glutathione $(\mathrm{GSH})$ to form $\mathrm{H}_{2} \mathrm{O}$ and oxidized glutathione (GSSG). If detection is carried out using $\mathrm{H}_{2} \mathrm{O}_{2}$ as a substrate, the enzymatic activity of catalase, which can also decompose $\mathrm{H}_{2} \mathrm{O}_{2}$, interferes with the measurement of GPx activity. We used an indirect assay here. The activity of GPx was determined by the speed of its enzymatic reaction and determined by the consumption of reduced GSH in this reaction. Moreover, the organic peroxide reagent $(\mathrm{t}-\mathrm{Bu}-\mathrm{OOH})$ provided in the kit does not react with GSH in the absence of GPx, nor is it affected by the intracellular catalase catalytic and decomposed. Therefore, GSH peroxidase activity can be detected more specifically. In addition, because the two substrates can undergo a redox reaction in the absence of an enzyme, the portion of the reduced GSH produced by this non-enzymatic reaction must be subtracted when enzyme activity is finally calculated.

Catalase activity was determined by initially stopping $\mathrm{H}_{2} \mathrm{O}_{2}$ decomposition by CAT with ammonium molybdate, followed by colorimetric (developed pale-yellow color) quantification of the remaining $\mathrm{H}_{2} \mathrm{O}_{2}$ and ammonium molybdate complex at $405 \mathrm{~nm}$ via spectrophotometry (Shimadzu UV-2550, Kyoto, Japan).

For colorimetric determination, various antioxidants in an organism can reduce $\mathrm{Fe}^{3+}$ to $\mathrm{Fe}^{2+}$. Furthermore, $\mathrm{Fe}^{2+}$ can form a stable complex with phenanthroline, which exhibits strong absorption at $520 \mathrm{~nm}$. Therefore, the level of antioxidant capacity can be measured colorimetrically.

\section{Quantitative of Total Protein}

Ground squirrels change from glucose metabolism to lipid metabolism during hibernation. Thus, as a glucose-metabolizing enzyme, GAPDH expression is modified and inhibited during hibernation. For example, Bell et al. (2014) reported that GAPDH expression in the skeletal muscles of Ictidomys tridecemlineatus 
differs significantly between torpor and interbout arousal in regard to post-translational modification, phosphorylation, acetylation, and methylation. In jerboas (Jaculus orientalis), liver GAPDH relies on post-translational modification to inhibit activity during hibernation and decreases in GAPDH expression in skeletal muscle inhibit GAPDH activity (Soukri et al., 1996). Therefore, GAPDH cannot be used as an internal control reference. In addition, other internal reference proteins, such as $\alpha$-tubulin, $\beta$-tubulin, and $\beta$-actin, are expressed differently in different tissues (Dittmer and Dittmer, 2006) and can (e.g., $\beta$-actin) vary with age in hibernators (Ferguson et al., 2005; Vigelso et al., 2015). However, total protein quantification can avoid these issues when used as a internal control in Western blot analysis (Aldridge et al., 2008; Ladner et al., 2004; Li and Shen, 2013). Therefore, we used total proteins in each group as the internal reference.

The electrophoresis separation gel was scanned with an electrophoresis gel imager (Syngene, United Kingdom), and the net optical density of the total proteins in each lane for all blots was determined using NIH Image J software.

\section{Western Blotting}

We detected specific proteins in the tissue homogenates using Western blotting, as described previously (Wei et al., 2018a,b). Total proteins were extracted from the skeletal muscle samples using RIPA lysis (Heart, Xi'an, China). The protein concentrations were measured using a Pierce ${ }^{\mathrm{TM}}$ bicinchoninic acid (BCA) protein quantitation kit (Thermo Fisher Scientific, Rockford, IL, United States), as per the provided instructions.

Equal volumes of proteins were used for $10 \%$ SDSPAGE gel and polyvinylidene difluoride membrane blotting, as per prior research (Yang et al., 2014). After $45 \mathrm{~min}$ of electrophoresis, all proteins were transferred using 25-mM Tris buffer solution $(\mathrm{pH}=8.5)$ to PVDF membranes $(0.45 \mu \mathrm{m})$, with the membranes then blocked for $2 \mathrm{~h}$ with $5 \%$ fat-free milk and finally decanted. The membranes were subjected to overnight incubation $\left(4^{\circ} \mathrm{C}\right)$ with specific primary antibodies for SOD1, SOD2, CAT, GPx1, Nrf2, Nrf2 (phospho S40), and Keap1 (as listed in Supplementary Table S2), followed by $2 \mathrm{~h}$ of incubation (room temperature) with HRP-linked antirabbit IgG secondary antibody (1:5000 v:v dilution, Thermo Fisher Scientific, United States) in TBST. The membranes were finally washed four times with TBST ( $5 \mathrm{~min} /$ wash) and visualized by enhanced chemiluminescence reagents (Thermo Fisher Scientific, United States) following the manufacturer's instructions. Band intensity was determined using NIH ImageJ software. Total protein stain was applied for normalization to determine protein abundance (i.e., proteins of interest were normalized to total proteins in each lane for all blots) (Zhang et al., 2019).

\section{Statistical Analysis}

Differences among experimental groups were assessed using oneway analysis of variance (ANOVA) and Fisher's LSD post hoc tests. In cases of no homogeneity, ANOVA and Dunnett's T3 tests were used. All statistical tests were conducted using SPSS v19.0 and a $P$-value of $<0.05$ was deemed significant.

\section{RESULTS}

\section{Changes in $\mathrm{H}_{2} \mathrm{O}_{2}$ and MDA Levels in Different Skeletal Muscles Over Torpor-Arousal Cycle}

As surrogate measures of oxidative stress and damage from free radicals, including ROS, we measured changes in MDA and $\mathrm{H}_{2} \mathrm{O}_{2}$ levels in the different skeletal muscles of hibernating ground squirrels (Figure 3).

Compared with the $\mathrm{SA}$ group, $\mathrm{H}_{2} \mathrm{O}_{2}$ content in the SOL showed no significant changes during the hibernation season. However, $\mathrm{H}_{2} \mathrm{O}_{2}$ levels in the EDL and GAS decreased significantly by $21.3 \%(P<0.05)$ and $29.9 \%(P<0.001)$, respectively, in the PRE group and by $21.4 \%(P<0.05)$ and $19.8 \%(P<0.01)$, respectively, in the LT group. In the POST group, $\mathrm{H}_{2} \mathrm{O}_{2}$ content recovered to SA levels in all three muscles (Figure 3A).

The MDA levels in all three muscles were markedly lower in the IBA and ET groups than in the SA and PRE groups. In the SOL, MDA levels in the LT group were significantly higher than those in the IBA and ET groups (204.8\%, $P<0.01$ and $233.1 \%$, $P<0.01$, respectively). However, MDA levels in the EDL and GAS muscles of the LT group decreased by $55.5 \%(P<0.05)$ and $75.2 \%(P<0.01)$, respectively, compared with the SA group. Similar to the changes in $\mathrm{H}_{2} \mathrm{O}_{2}$ levels, MDA content in all muscles recovered to normal levels after hibernation (Figure 3B).

\section{Expression of Antioxidant Proteins in Different Skeletal Muscles Over Torpor-Arousal Cycle}

The proteins of interest were normalized to total proteins (Zhang et al., 2019). Figure 4 shows the total protein gel images. In regard to antioxidant defense, we performed Western blot analysis to ascertain changes in the expression levels of several antioxidant proteins in the SOL, EDL, and GAS muscles, as shown in Figure 5.

Compared with the SA group, SOD1 expression in the SOL of the IBA and POST groups increased significantly (45.4 and $39.9 \%, P<0.05$, respectively). Furthermore, GPx1 expression remained at a higher level through the course of hibernation relative to the SA group, and increased by $81.0 \%(P<0.01), 82.1 \%$ $(P<0.01)$, 51.8\% $(P<0.05)$, and $64.7 \%(P<0.05)$ in the IBA, ET, LT, and POST groups, respectively. SOD2 levels in the IBA group increased by $54.7 \%(P<0.05)$. CAT protein remained at a high level throughout the hibernation season relative to the SA group, and increased by 30.9\% $(P<0.05), 43.5 \%(P<0.01)$, 45.9\% $(P<0.01), 31.5 \%(P<0.05)$, and 34.2\% $(P<0.05)$ in the PRE, IBA, ET, LT, and POST groups, respectively. In the POST group, SOD1, GPx1, and CAT expression were higher, whereas SOD2 expression was similar to SA and PRE levels (Figures 5A,B).

Relative to the SA group, the SOD1 protein remained relatively stable in the EDL during hibernation. Furthermore, in the EDL, GPx1 expression increased significantly in the ET and LT groups $(32.8 \%, P<0.01 ; 25.0 \%, P<0.05$, respectively); 


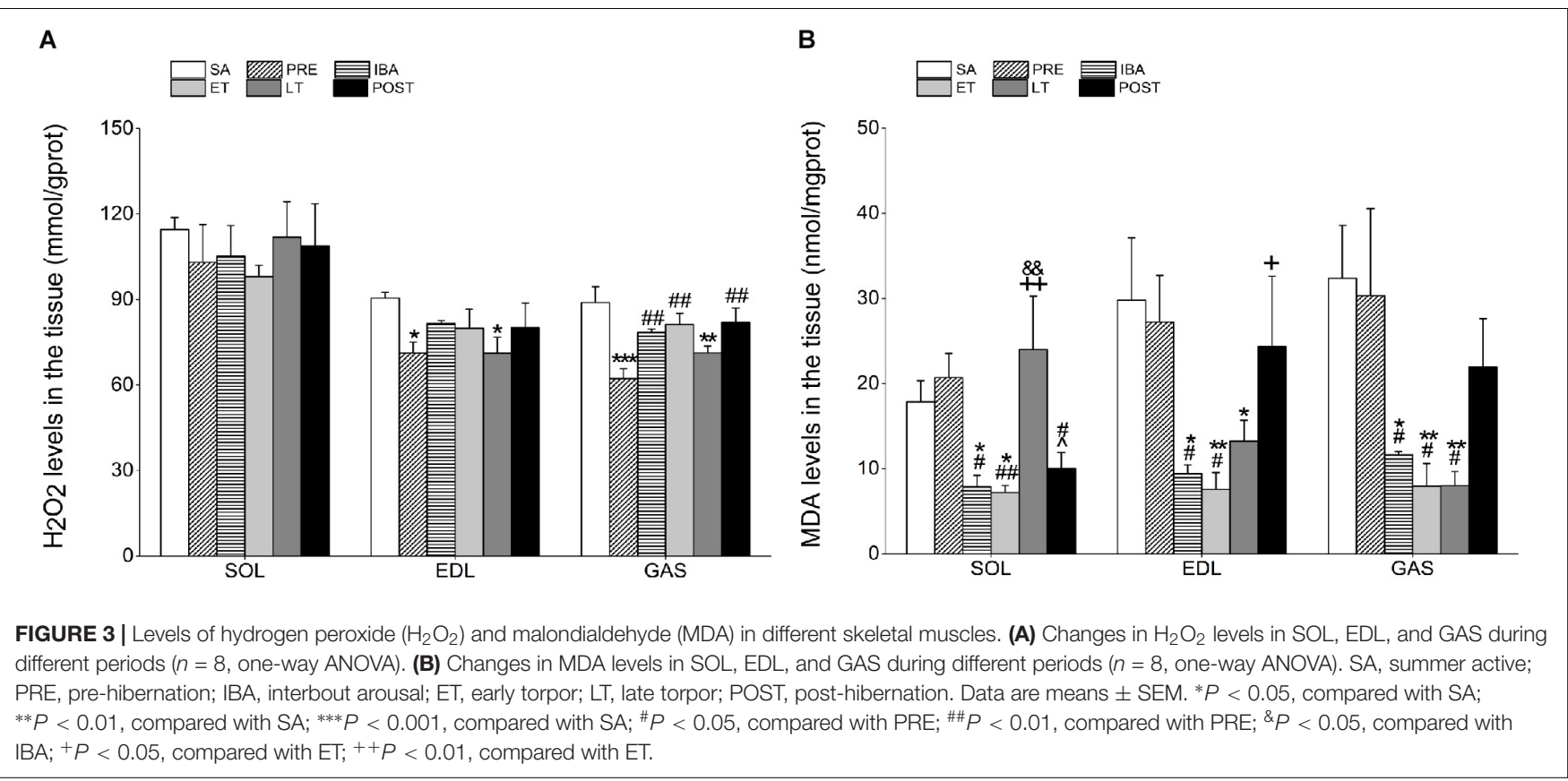

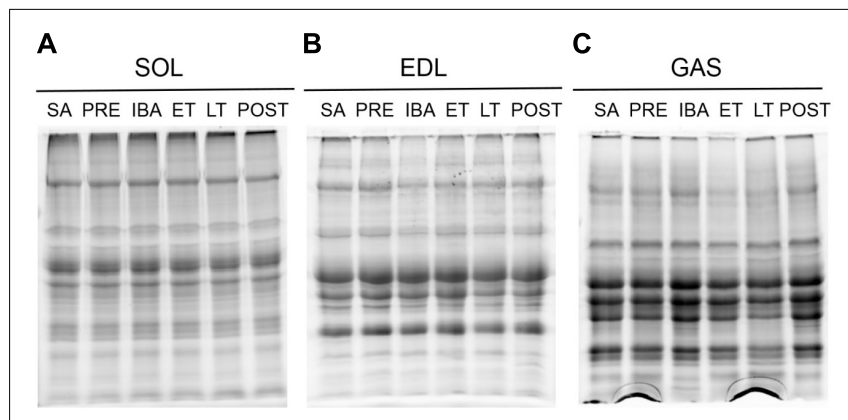

FIGURE 4 | Stain-free imaging of separation gel. (A) In the SOL. (B) In the EDL. (C) In the GAS. SOL, soleus; EDL, extensor digitorum longus; GAS, gastrocnemius. SA, summer active; PRE, pre-hibernation; IBA, interbout arousal; ET, early torpor; LT, late torpor; POST, post-hibernation.

SOD2 expression increased significantly in the IBA and POST groups $(26.0 \%, P<0.05 ; 64.9 \%, P<0.001$, respectively); and CAT expression increased dramatically in the ET, LT, and POST groups $(32.8 \%, P<0.05$; $53.0 \%, P<0.001 ; 86.0 \%, P<0.001$, respectively). In the POST group, SOD2 and CAT expression levels were higher, whereas SOD1 and GPx1 expression levels were similar to SA and PRE levels (Figures 5C,D).

In the GAS, SOD1 protein in the ET and POST groups increased markedly relative to the SA group $(25.0 \%, P<0.05$; $39.1 \%, P<0.001$, respectively). In addition, SOD2 was at higher levels throughout the entire hibernation period, and increased by $54.3 \%(P<0.05), 113.2 \%(P<0.05), 100.6 \%$ $(P<0.05)$, 99.4\% $(P<0.05)$, and $98.4 \%(P<0.05)$ in the PRE, IBA, ET, LT, and POST squirrels, respectively. GPx1 and CAT expression in the GAS remained relatively steady during hibernation (Figures 5E,F).

\section{Antioxidant Enzyme Activity in Different Skeletal Muscles Over Torpor-Arousal Cycle}

The activities of four antioxidant enzymes and TAC were determined to clarify their effects on oxidative stress, as shown in Figure 6.

Compared with the SA, PRE, IBA, ET, and LT groups, SOD1 activity in the SOL of the POST group increased significantly by $32.1 \%(P<0.01), 27.7 \%(P<0.05), 31.7 \%(P<0.01)$, 35.9\% $(P<0.01)$, and $21.3 \%(P<0.05)$, respectively. Relative to the SA group, GPx activity increased significantly in the IBA and POST groups (130.1\% and $153.8 \%, P<0.01$, respectively). Furthermore, SOD2 activity showed no obvious change in the SOL during the torpor-arousal cycle, whereas CAT activity increased markedly by $79.6 \%(P<0.05), 92.2 \%(P<0.05), 99.9 \%$ $(P<0.01)$, and $83.6 \%(P<0.05)$ in the IBA, ET, LT and POST, respectively. The TAC in the SOL increased by $43.1 \%(P<0.05)$ in the POST group compared with the PRE group (Figure 6A).

Compared with the SA group, SOD1 activity in the EDL decreased significantly by $18.8 \%(P<0.05)$ in the PRE group, but recovered to SA levels in the POST group. GPx activity in the EDL did not show a clear change during hibernation, whereas SOD2 activity decreased throughout the hibernation season, with a significant decrease of $45.2 \%(P<0.05)$ in the ET group. However, relative to the SA group, CAT activity in the EDL showed significant increases of $70.3 \%(P<0.01)$, 97.0\% $(P<0.001)$, 58.3\% $(P<0.05)$, and 74.8\% $(P<0.01)$ in the IBA, ET, LT and POST groups, respectively. The TAC in the EDL showed a slight but non-significant increase during hibernation (Figure 6B).

Compared with the SA group, SOD1 activity in the GAS increased throughout the hibernation season, with a significant increase of $20.7 \%(P<0.05)$ in the POST group. Furthermore, 
A

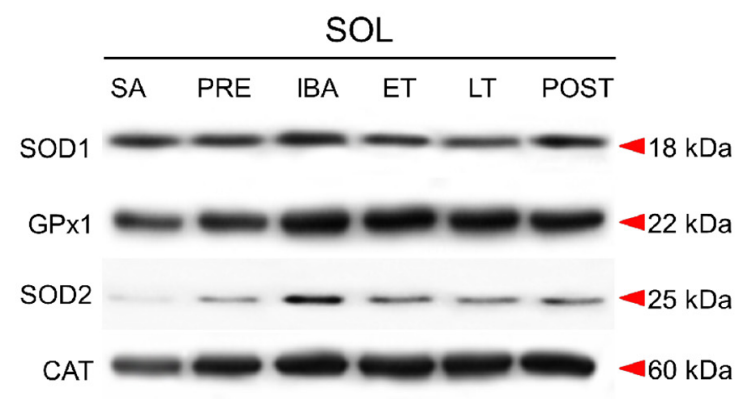

C

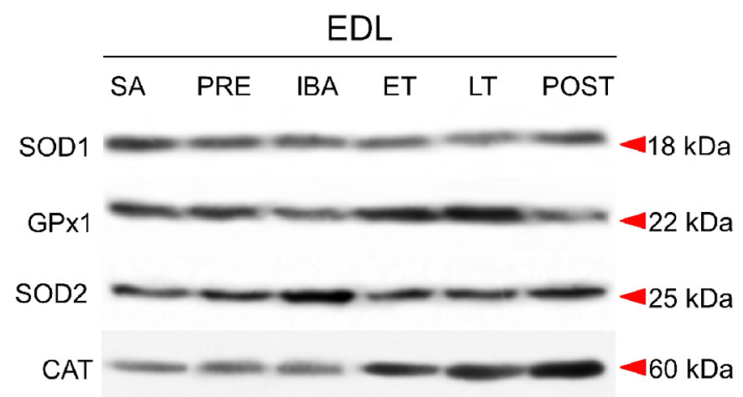

E

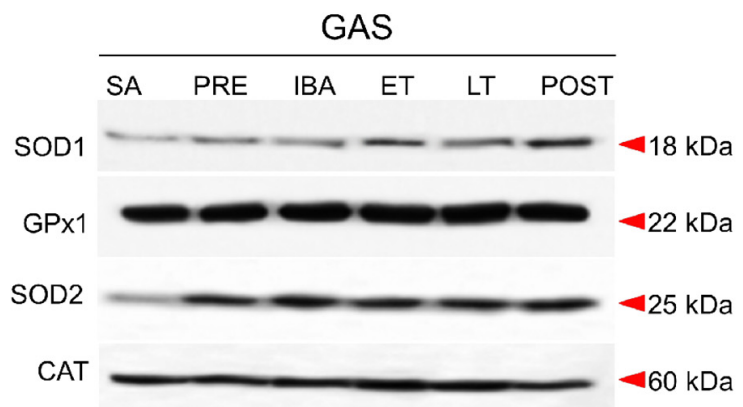

B

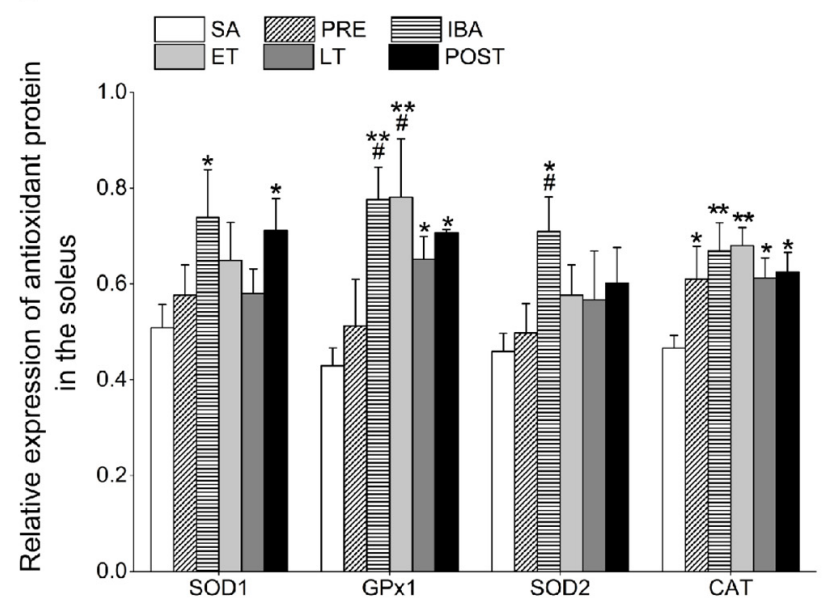

D

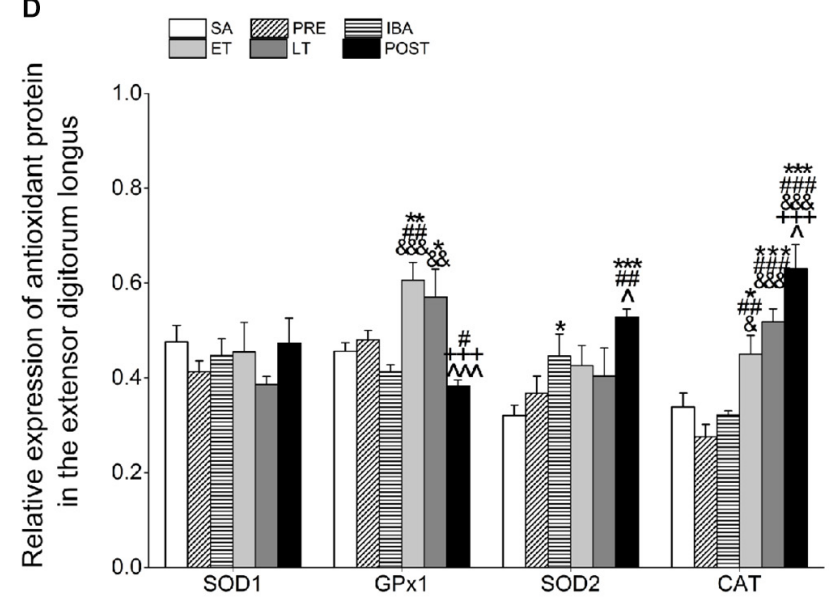

$\mathbf{F}$

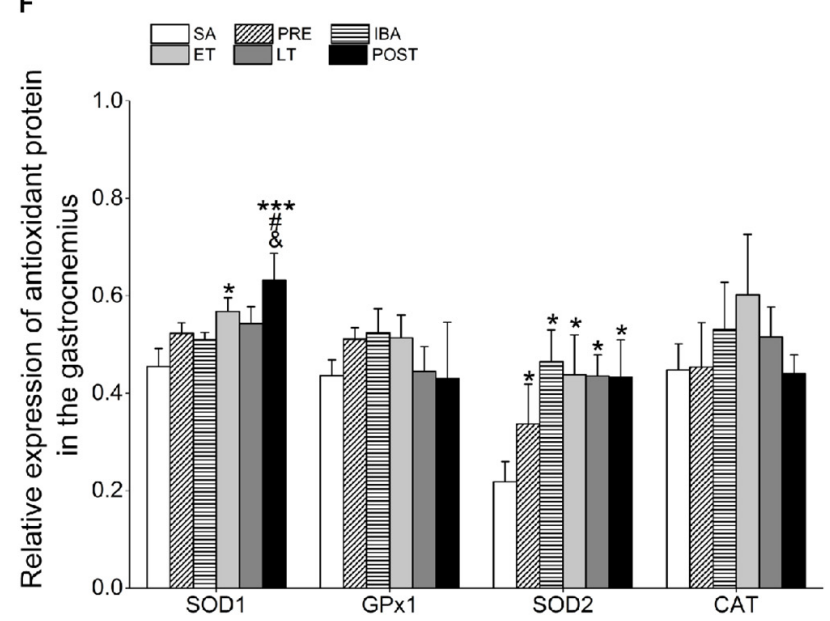

FIGURE 5 | Changes in levels of SOD1, GPX1, SOD2, and CAT proteins in different skeletal muscles of Daurian ground squirrels over the torpor-arousal cycle. (A) Representative immunoblots of SOD1, GPx1, SOD2, and CAT in SOL during six hibernation periods. (B) Relative SOD1, GPx1, SOD2, and CAT protein expression in SOL. (C) Representative immunoblots of SOD1, GPx1, SOD2, and CAT in EDL during six hibernation periods. (D) Relative SOD1, GPx1, SOD2, and CAT protein expression in EDL. (E) Representative immunoblots of SOD1, GPx1, SOD2, and CAT in GAS during six hibernation periods. (F) Relative SOD1, GPx1, SOD2, and CAT protein expression in GAS. SA, summer active; PRE, pre-hibernation; IBA, interbout arousal; ET, early torpor; LT, late torpor; POST, post-hibernation. Values are means \pm SEM, $n=8$. ${ }^{*} P<0.05$, Compared with SA; ${ }^{* *} P<0.01$, compared with SA; ${ }^{* * *} P<0.001$, compared with SA; ${ }^{*} P<0.05$, compared with PRE; $\# \#<0.01$, compared with PRE; \#\# $P<0.001$, compared with PRE; \& $P<0.05$, compared with IBA; \&\& $P<0.01$, compared with IBA; \&\&\& $P<0.001$, compared with IBA; ${ }^{+++} P<0.001$, compared with $\mathrm{ET} ; \wedge P<0.05$, compared with $\mathrm{LT} ; \wedge \wedge \wedge P<0.01$, compared with $\mathrm{LT}$. 

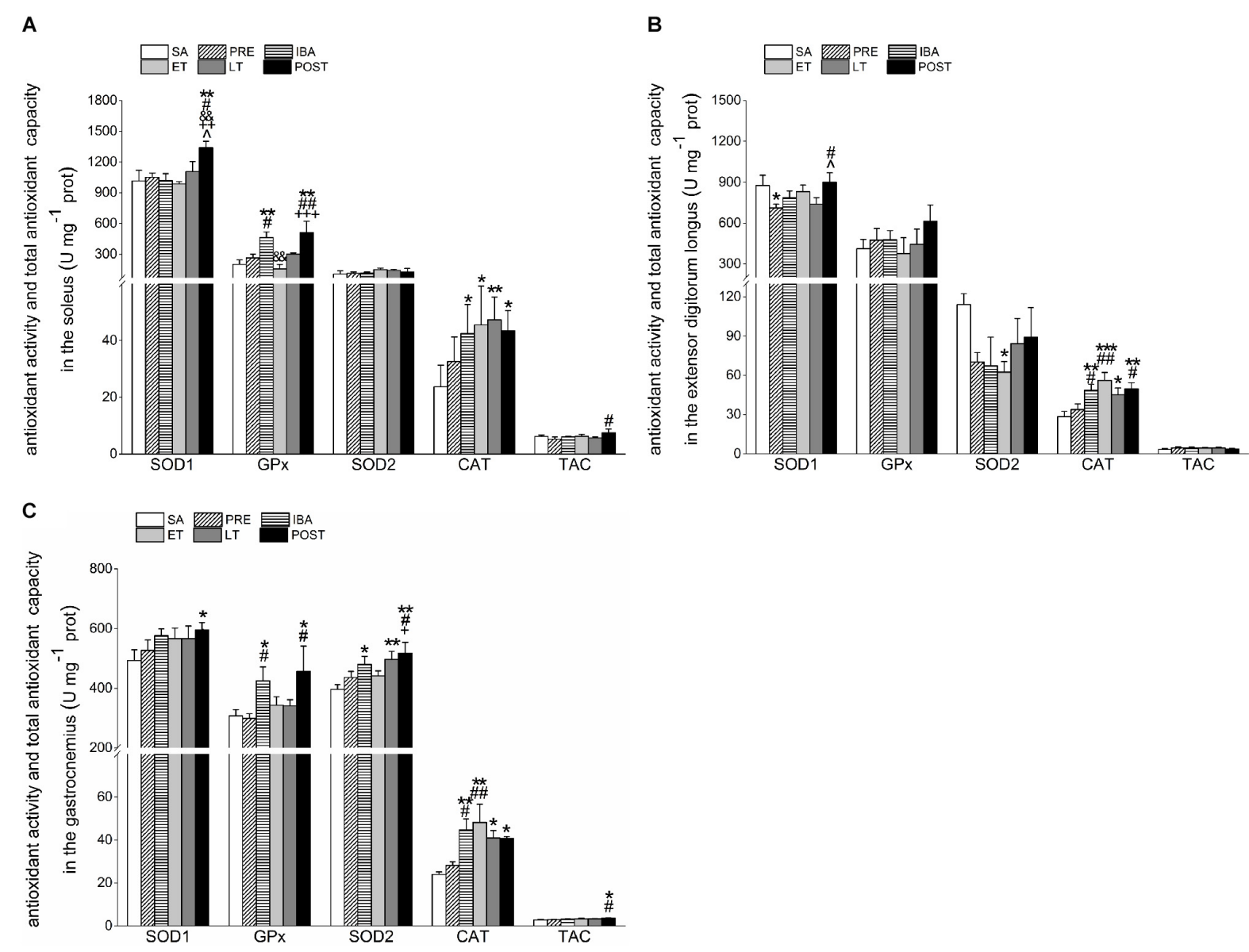

FIGURE 6 | Changes in activities of SOD1, SOD2, CAT, GPX1, and TAC in different skeletal muscles of Daurian ground squirrels over the torpor-arousal cycle. (A) Activities of SOD1, SOD2, CAT, GPx, and TAC in SOL during six hibernation periods. (B) Activities of SOD1, SOD2, CAT, GPx, and TAC in EDL during six hibernation periods. (C) Activities of SOD1, SOD2, CAT, GPx, and TAC in GAS during six hibernation periods. SA, summer active; PRE, pre-hibernation; IBA, interbout arousal; ET, early torpor; LT, late torpor; POST, post-hibernation; SOL, soleus; EDL, extensor digitorum longus; GAS, gastrocnemius. Values are means \pm SEM, $n=8$. ${ }^{*} P<0.05$, Compared with SA; ${ }^{* *} P<0.01$, compared with SA; ${ }^{* * *} P<0.001$, compared with SA; ${ }^{*} P<0.05$, compared with PRE; $\# \#<0.01$, compared with PRE; \&\& $P<0.01$, compared with IBA; ${ }^{+} P<0.05$, compared with $\mathrm{ET}$; ${ }^{++} P<0.01$, compared with $\mathrm{ET}$; ${ }^{+++} P<0.001$, compared with $\mathrm{ET} ;{ }^{\wedge} P<0.05$, compared with LT.

GPx activity in the GAS increased significantly by $38.2 \%$ $(P<0.05)$ and $48.2 \%(P<0.05)$ in the IBA and POST groups, respectively, and SOD2 activity increased significantly by $21.2 \%$ $(P<0.05), 25.5 \%(P<0.01)$, and 30.7\% $(P<0.01)$ in the IBA, LT, and POST groups, respectively. Relative to the SA group, CAT activity in the GAS increased considerably by $87.0 \%$ $(P<0.01), 101.5 \%(P<0.01), 71.1 \%(P<0.05)$, and $70.2 \%$ $(P<0.05)$ in the IBA, ET, LT, and POST, respectively. In addition, TAC in the GAS increased during the hibernation season, and increased markedly by $32.6 \%(P<0.05)$ and $27.2 \%(P<0.05)$ in the POST group compared with the SA and PRE groups, respectively (Figure 6C).

\section{Regulation of Antioxidant Transcription in Different Skeletal Muscles}

The Nrf2/Keap1 signaling pathway is crucial for antioxidant defense regulation in hibernating mammals (Yin et al., 2016). In consideration of the effects of oxidative stress on this pathway during the torpor-arousal cycle, we measured Nrf2, p-Nrf2 (phosphorylated Nrf2), and Keap1 expression in skeletal muscles via Western blot analysis (Figure 7).

Compared with the SA group, the Nrf2 protein level in the SOL increased significantly by $25.7 \%(P<0.05), 23.6 \%$ $(P<0.05)$, 46.2\% $(P<0.001)$, and $28.6 \%(P<0.05)$ in the IBA, ET, LT, and POST groups, respectively. Furthermore, relative to the SA group, the expression of $\mathrm{p}-\mathrm{Nrf} 2$ in the SOL increased markedly by $27.8 \%(P<0.05), 32.6 \%(P<0.05)$, and $32.7 \%$ $(P<0.05)$ in the IBA, LT, and POST groups, respectively. Keap1 expression in the SOL increased by $31.8 \%(P<0.05)$ and $27.1 \%(P<0.05)$ in the PRE and IBA groups, respectively (Figures 7A,B).

Compared with the SA group, the Nrf2 protein level in the EDL increased significantly by $89.3 \%(P<0.01), 94.7 \%$ $(P<0.01), 81.9 \%(P<0.01)$, and $83.3 \%(P<0.01)$ in the IBA, ET, LT, and POST groups, respectively. Furthermore, compared 
A

SOL

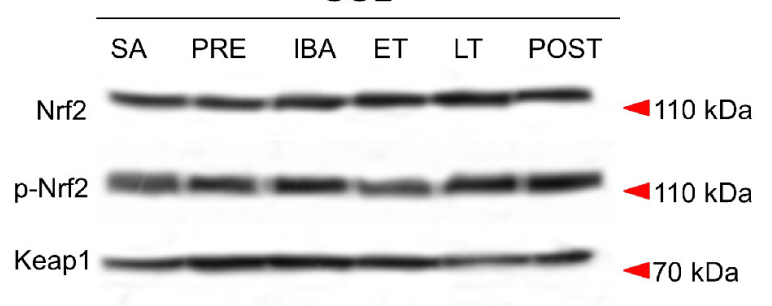

C

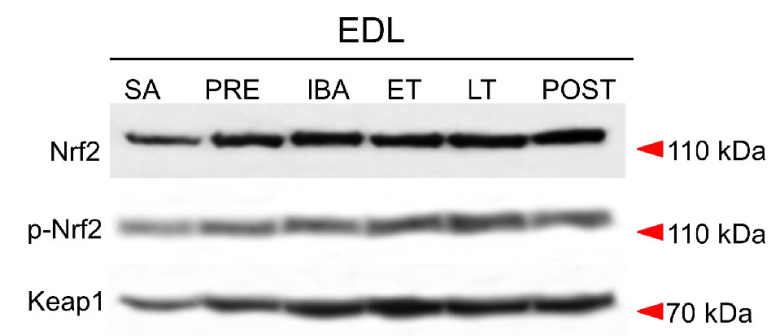

E

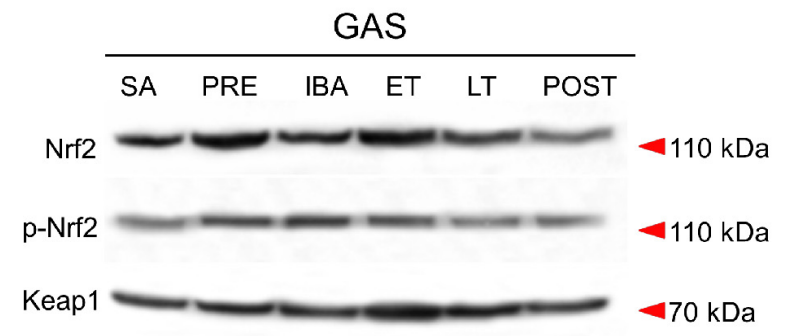

B

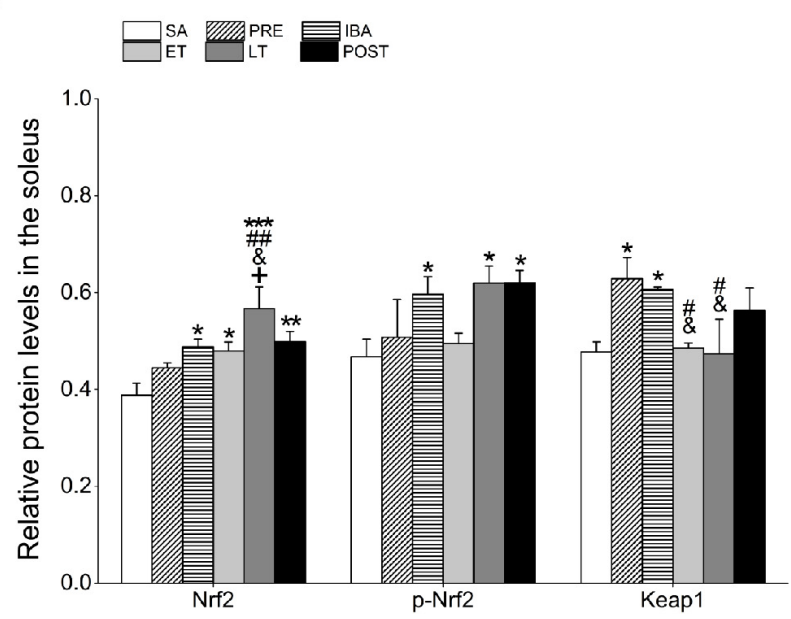

D

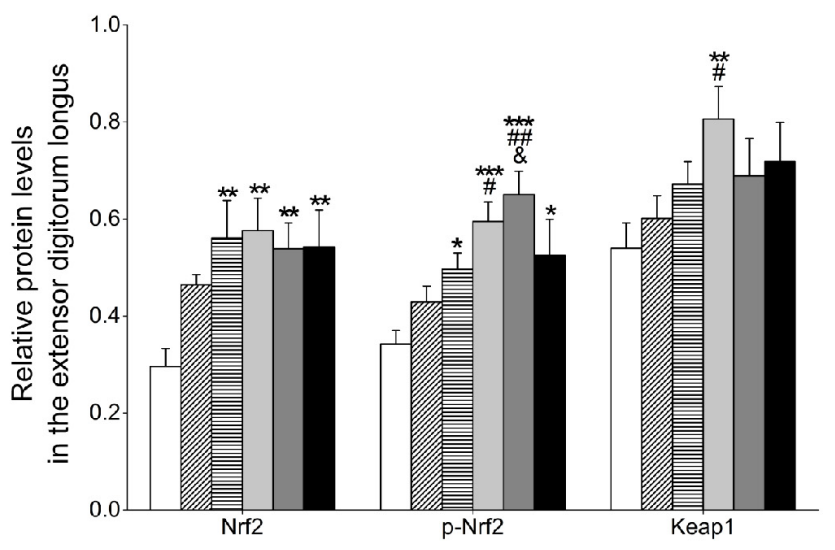

$\mathbf{F}$

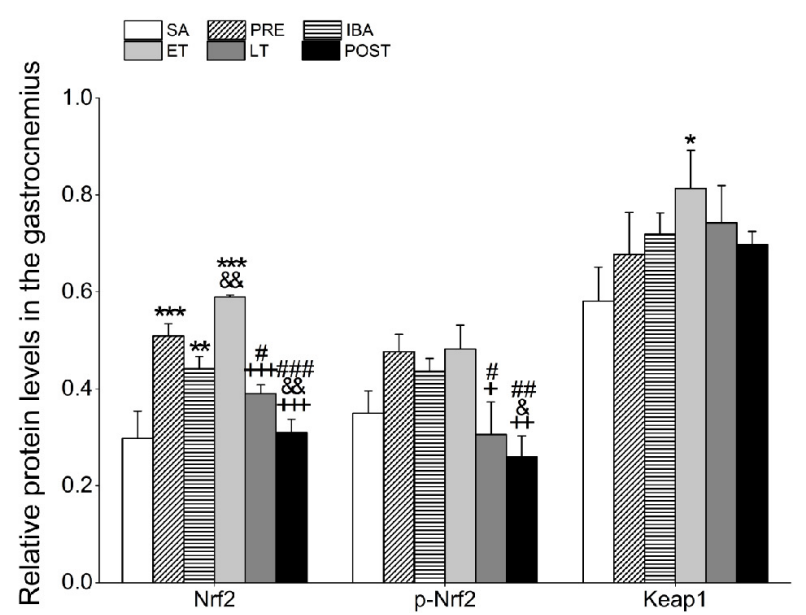

FIGURE 7 | Changes in Nrf2, p-Nrf2, and Keap1 protein levels in different skeletal muscles of Daurian ground squirrels over the torpor-arousal cycle. (A) Representative immunoblots of Nrf2, p-Nrf2, and Keap1 in SOL during six hibernation periods. (B) Relative Nrf2, p-Nrf2, and Keap1 protein expression in SOL. (C) Representative immunoblots of Nrf2, p-Nrf2, and Keap1 in EDL during six hibernation periods. (D) Relative Nrf2, p-Nrf2, and Keap1 protein expression in EDL. (E) Representative immunoblots of Nrf2, p-Nrf2, and Keap1 in GAS during six hibernation periods. (F) Relative Nrf2, p-Nrf2, and Keap1 protein expression in GAS. SA, summer active; PRE, pre-hibernation; IBA, interbout arousal; ET, early torpor; LT, late torpor; POST, post-hibernation. Values are means $\pm \mathrm{SEM}, n=8 .{ }^{*} P<0.05$, Compared with SA; ${ }^{* *} P<0.01$, compared with SA; ${ }^{* * *} P<0.01$, compared with SA; ${ }^{*} P<0.05$, compared with PRE; $\#$ \# $P$.01, compared with PRE;

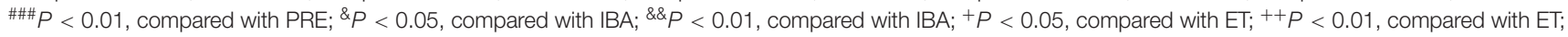
$+++P<0.001$, compared with ET. 
with the SA group, the p-Nrf2 expression in the EDL increased markedly by $45.4 \%(P<0.05), 73.9 \%(P<0.001), 90.1 \%$ $(P<0.001)$, and $21.3 \%(P<0.05)$ in the IBA, ET, LT, and POST groups, respectively, and Keap1 expression increased by $49.5 \%$ $(P<0.01)$ in the ET group (Figures 7C,D).

Compared with the SA group, Nrf2 expression in the GAS increased significantly by $71.0 \%(P<0.001), 48.6 \%(P<0.01)$, and $98.1 \%(P<0.01)$ in the PRE, IBA, and ET groups, respectively. The p-Nrf2 expression in the GAS showed a slight increase in the PRE, IBA and ET groups, whereas Keap1 expression showed a significant rising trend during hibernation, with a marked increase of $40.0 \%(P<0.05)$ in the ET relative to the SA group (Figures 7E,F).

\section{DISCUSSION}

This study is the first to report on the remarkable protective effects of Nrf2-mediated antioxidant enzymes and tissue specificity in different skeletal muscles over the torpor-arousal cycle in Daurian ground squirrels. Results demonstrated that most tested indicators recovered to summer or pre-hibernation levels following their modification (increase or decrease) during hibernation, thus suggesting exceptional redox homeostasis. Moreover, the Nrf2/Keap1 signaling pathway was activated over the torpor-arousal period in the three skeletal muscles, which, in turn, up-regulated the expression of various antioxidant enzymes. These results indicated that the enhanced antioxidation defense ability mediated by the Nrf2/Keap1 pathway during repeated ischemia-reperfusion in skeletal muscles may be a vital mechanism to protect against oxidative damage during hibernation.

$\mathrm{H}_{2} \mathrm{O}_{2}$ is a significant ROS, and its increased production or failed breakdown can reflect increased oxidative stress (Halliwell and Cross, 1994; Turrens, 2003; Chainy et al., 2016). In the current study, $\mathrm{H}_{2} \mathrm{O}_{2}$ content in the EDL and GAS decreased significantly in the PRE and LT states. Pre-hibernation is a stage during which animals prepare for successful hibernation. For example, many hibernators will fatten extensively before the hibernation season to build-up fat stores that are relied on as an energy source during winter, or do not hibernate when lean (Geiser, 2013). Therefore, we speculated that this phenomenon may be the result of a forward-looking downregulation of the oxidant system to cope with the multiple oxidative stresses experienced during hibernation; however, the specific mechanism is still unknown. Entrance into late torpor results in the transitory interruption of euthermic conditions, and includes considerable reductions in heart rate, blood flow, oxygen consumption, and mitochondrial respiration (Carey et al., 2003). For example, previous research has reported reductions in oxygen uptake of $>90 \%$ in Arctic ground squirrels during late stage hibernation (Toien et al., 2001). Although animals face substantial stress during hibernation, including hypothermia, hypoxia, and ischemia, we found that $\mathrm{H}_{2} \mathrm{O}_{2}$ levels were decreased significantly in the LT group. This is similar to earlier studies on the visceral tissues of hibernators. For instance, Wei et al. (2018b) reported that
$\mathrm{H}_{2} \mathrm{O}_{2}$ levels in the liver and kidneys of Daurian ground squirrels do not increase during hibernation. In addition, Yin et al. (2016), who studied the brain tissues of hibernating bats (Myotis ricketti and Rhinolophus ferrumequinum), demonstrated that ROS levels are lower during torpor than during the active state. In the current study, the significant decrease in $\mathrm{H}_{2} \mathrm{O}_{2}$ content in the EDL and GAS muscles during torpor suggests that oxidative stress did not occur in the skeletal muscles during hibernation, despite the stressful conditions. However, unlike the EDL and GAS, $\mathrm{H}_{2} \mathrm{O}_{2}$ levels in the SOL were slightly elevated in the LT state. One reason for this distinction may be the different proportions of muscle fiber type. The antigravity slow-twitch SOL muscle, which consists predominantly of MHC I oxidative fibers, contains a large number of mitochondria and relies on aerobic oxidation to generate energy. Conversely, fast-twitch EDL and mixed GAS muscles, which consist of MHC II glycolytic fibers or a mix of oxidative and glycolytic fibers, contain fewer mitochondria and rely on anaerobic glycolysis to provide energy. As mitochondria are the major sites of ROS generation, this may explain the higher levels of $\mathrm{H}_{2} \mathrm{O}_{2}$ found in the SOL during the LT state compared with that found in the EDL and GAS. In conclusion, the predominant decrease in ROS levels in the skeletal muscles during hibernation indicates that hibernating ground squirrels avoided oxidative stress, instead demonstrating remarkable redox homeostasis ability under high stress environments.

As a bioindicator of oxidative damage, MDA can indirectly reflect the levels of both oxidative stress and damage. In the present study, MDA content in the SOL increased markedly in the LT state compared with that in the IBA state. For successful hibernation, stored polyunsaturated fatty acid (PUFA) content is elevated in lipids in order to preserve lipid fluidity under low Tb conditions (Frank, 1992). However, due to their carbon-carbon double bonds, PUFAs are highly prone to free radical attack and are readily oxidized, resulting in lipid peroxide radical generation (Gunstone, 1996), and here in the observed MDA increase in the SOL during hibernation. The SOL muscle is mitochondrially rich and reliant on aerobic oxidation, which is significant given the importance of the mitochondrial electron respiratory chain in ROS production. Thus, compared to other skeletal muscles, the SOL is more sensitive to ROS via hypoxic ischemia-reperfusion. This is supported by previous study on Daurian ground squirrels, which showed that MDA content increases significantly during late torpor in tissues with the highest blood flow and oxygen consumption (i.e., heart and brain) (Wei et al., 2018b). In contrast, however, we observed significantly lower MDA content in the EDL and GAS muscles during LT than during the SA state. This indicated that under multiple stress conditions, the EDL and GAS not only avoided oxidative stress during hibernation, but the levels were lower than that observed under normal SA conditions. Moreover, MDA content in the IBA state was markedly lower than levels observed in the SA state in all three muscles, suggesting that IBA is an important period for hibernating animals to down-regulate ROS and prevent hibernation-induced oxidative stress. Under certain 
stress conditions, ROS production can become unregulated, resulting in the oxidization of various biomolecules, such as carbohydrates, lipids, and DNA, as well as the impairment of cellular functions and the promotion of cellular death (EspinosaDiez et al., 2015). In contrast, under normal conditions, ROS play significant physiological roles as essential "second messengers" and are vital in intracellular signaling and regulation (Valko et al., 2007; Circu and Aw, 2010). This, together with the relatively low $\mathrm{H}_{2} \mathrm{O}_{2}$ levels observed during hibernation in our study, suggests that ground squirrels maintain a basal level of ROS during hibernation that retains function but does not harm tissue.

Shivering thermogenesis in skeletal muscle, which helps return $\mathrm{Tb}$ to euthermic levels, can actually trigger oxidative stress due to blood oxygen reperfusion and subsequent production of ROS (Barja de Quiroga, 1992; Dark and Miller, 1997). As oxidative stress is associated with both an increase in the production of oxidizing species and a decrease in antioxidant defense capability within the body, we detected changes in the protein expression of multiple antioxidants (i.e., SOD1, SOD2, CAT, and GPx1) in skeletal muscles under various stages of hibernation. In the SOL, the SOD1 protein levels in the IBA and POST states and SOD2 protein level in the IBA state increased markedly. The initiation of IBA is associated with the resumption of those physiological functions suppressed during torpor, including the rapid increase in $\mathrm{Tb}\left(35-38^{\circ} \mathrm{C}\right)$ and tissue reperfusion of blood and oxygen. Furthermore, at the completion of hibernation, animals experience a return of normal physiological function (Carey et al., 2003; Storey, 2010). Previous research on thirteen-lined ground squirrels has shown that oxygen consumption is significantly higher at arousal than during torpor (Muleme et al., 2006) and liver mitochondrial respiration rapidly increases following its suppression during hibernation (Mathers et al., 2017). Here, the significantly increased SOD1 and SOD2 expression levels observed in the IBA and POST groups suggest a need for SOD proteins in the SOL during the rewarming period when $\mathrm{Tb}$ recovers to euthermic levels. However, GPx1 and CAT expression levels in the SOL increased dramatically throughout the hibernation season, suggesting that these two antioxidant enzymes may contribute to improved antioxidant defense and protect cells for long periods during hypometabolism. Based on proteomic analysis, previous research on the skeletal muscles of Daurian ground squirrels found that SOD1 and CAT increase significantly in post-hibernation (Chang et al., 2016). SOD1 catalyzes the dismutation of $\mathrm{O}_{2}{ }^{-}$.and eliminates free radicals harmful to biological systems, whereas CAT decomposes $\mathrm{H}_{2} \mathrm{O}_{2}$ into $\mathrm{H}_{2} \mathrm{O}$ and protects cells from $\mathrm{H}_{2} \mathrm{O}_{2}$ toxicity (Halliwell and Cross, 1994; Chainy et al., 2016). As a slow-twitch muscle rich in mitochondria and dependent on aerobic oxidation (Peter et al., 1972), the SOL appears to be protected against potential oxidative stress-induced damage by the up-regulation of antioxidant enzymes. In the EDL, GPx1 and CAT expression during ET and LT increased markedly. Compared with levels under normal euthermic conditions, many physiological functions undergo temporary suspension or reduction during torpor (ET and LT), including substantial falls in metabolic rate (to $1-5 \%$ ), $\mathrm{Tb}$ (as low as $1-5^{\circ} \mathrm{C}$ ), blood flow, and oxygen uptake (Geiser, 2004; Storey, 2010). In the current study, however, under conditions of fasting and physiological inhibition, the expression of CAT and GPx1 were up-regulated, further suggesting the importance of enhanced antioxidant defense in hibernators to cope with and protect cells in high stress environments. In contrast, the SOD2 level increased substantially in the IBA and POST groups, implying that SOD2 may play a major role during IBA with high consumption of oxygen. Previous studies have shown that CAT, GPx, and SOD1 increase significantly and SOD2 decreases significantly in the skeletal muscles (quadriceps, fasttwitch muscle) of European ground squirrels during torpor (Vucetic et al., 2013). However, the protein expression of SODs in the present study is inconsistent with that reported in quadriceps, which may be due to the different effects of muscle mitochondrial productivity and energy metabolism on organisms. In the GAS, SOD1 protein expression increased significantly in the ET and POST groups, with SOD2 expression remaining at a high level throughout the hibernation season, and GPx1 and CAT remaining unchanged. These results suggest that SODs play a vital role in protecting against oxidative damage in the GAS. Earlier research has reported that TAC in the GAS is higher during torpor (156\%) than in summer-active animals, suggesting a potential mechanism for maintenance of muscle performance (James et al., 2013). Here, the activity of antioxidant enzymes also increased in the IBA and POST groups compared to the LT group in all three skeletal muscles. We speculate that this may be caused by the accelerated blood flow velocity and recovery of normal physiological indicators during the IBA and POST states than that during the LT state. In addition, markedly increased CAT activity was observed over the hibernation season in all three muscles. Thus, this increase in activity may be a common skeletal muscle mechanism in hibernators to prevent oxidative injury due to repeated ischemiareperfusion and prolonged muscle disuse over the torpor-arousal cycle. Nevertheless, in the present study, SOD2 activity showed different changes in the three skeletal muscles. Specifically, SOD2 activity increased significantly in various periods (IBA, LT, and POST) in the GAS, but showed no significant changes in the SOL and decreased in the EDL. Thus, the importance of SOD in the three skeletal muscles may differ. SOD2 activity was only elevated in the GAS, suggesting its importance for antioxidant capacity in acute muscle performance. However, the specific mechanism involved needs further study. During torpor, an animal experiences marked, though temporary, suppression in oxygen delivery and mitochondrial respiration; however, both are promptly restored during interbout arousal and accompanied by rapid tissue reperfusion (Carey et al., 2003; Storey, 2010). In response to ischemia-reperfusion, adequate antioxidant defense is needed to sustain cell viability over weeks of torpor and to defend against potential cellular stress during the transition stages. Indeed, our data showed that the protein expression and activity of four antioxidants increased significantly during hibernation bouts. Earlier studies have also demonstrated that various small molecular weight antioxidants and enzymes increase dramatically during torpor (Eddy et al., 2005; Ohta et al., 2006; Okamoto et al., 2006; 
Morin and Storey, 2007; Morin et al., 2008; Rouble et al., 2014; Yin et al., 2016; Wei et al., 2018b). For example, Vucetic et al. (2013) and Wei et al. (2018b) reported significant increases in SOD, CAT, and GPx1 protein and activity levels in the heart, liver, brain, and kidney tissues of Daurian ground squirrels and in the quadricep tissues of European ground squirrels, respectively, during hibernation. Allan and Storey (2012) found significant increases in SOD2 protein expression in mixed skeletal muscles during early hibernation, which remained high throughout, and Wu and Storey (2014) also found increases in CAT mRNA expression in the skeletal muscles of thirteenlined ground squirrels. In conclusion, based on our observed results, the significant decrease in $\mathrm{H}_{2} \mathrm{O}_{2}$ and $\mathrm{MDA}$ levels during hibernation may be due to the decomposition and elimination of excess ROS following the up-regulation of antioxidant protein expression and activity, thus suggesting the importance of this up-regulation in preventing skeletal muscle oxidative damage. In addition, the expression of different antioxidant enzymes showed muscle tissue specificity. This may be due to different oxygen consumption and sensitivity to oxidants as well as the physiological state of different muscles during hibernation. Overall, among the three muscles, antioxidant enzymes were up-regulated at different stages of hibernation, indicating that the skeletal muscle of Daurian ground squirrels can control oxidative stress induced by repeated ischemia-reperfusion and skeletal muscle disuse.

Thus, hibernators appear to possess a well-developed antioxidant defense system. Antioxidant responses can be promoted in a variety of ways, including activation of gene regulating pathways. For example, the Nrf2/Keap1 antioxidant pathway plays a critical role in cytoprotective responses to oxidative stress (Motohashi and Yamamoto, 2004; Baird and Dinkova-Kostova, 2011). Thus, we further examined the expression of the Nrf2/Keap1 antioxidant pathway in the different skeletal muscles. Results showed the Nrf2 and p-Nrf2 protein levels increased significantly in the SOL and EDL throughout the hibernation season, and dramatically increased in the GAS during the PRE, IBA, and ET states. These results are similar to those found in studies on adipose, heart, liver, brain, and kidney tissues in hibernators. For example, $\mathrm{Xu}$ et al. (2013) reported that Nrf2 protein expression is significantly higher in the skeletal muscle (Xu et al., 2013), brown adipose tissue, heart, and liver of 13lined ground squirrels (Morin et al., 2008) during hibernation than during the summer-active state. Ni and Storey (2010) demonstrated that significant increases in Nrf2 transcription factor expression levels in adipose tissues of hibernating squirrels during torpor and arousal can provide enhanced antioxidant defense to counter oxidative stress. Our previous research also showed increased Nrf2 and p-Nrf2 protein levels in the heart, liver, brain, and kidney tissue of hibernating Daurian ground squirrels (Wei et al., 2018a). The Nrf2/Keap1 signaling pathway is activated during oxidative stress, which results in the release of $\mathrm{Nrf} 2$ from Keap1 and its translocation into the nucleus. Nrf2 and small Maf proteins then undergo heterodimerization and antioxidant response element (ARE)binding in the nucleus, with the subsequent activation of various cytoprotective genes (Sun et al., 2007; EspinosaDiez et al., 2015). In our study, the significant increase in Nrf2 protein during torpor suggests that the elevated levels of antioxidant proteins (SODs, CAT, GPx) observed in the skeletal muscles during hibernation may be achieved through activation of the Nrf2 signaling pathway. Therefore, enhancing antioxidant defense ability by up-regulating the Nrf2/Keap1 antioxidant signaling pathway may be a common mechanism for hibernators to protect skeletal muscle and major visceral tissues from oxidative stress and may be triggered as one of the first events after entering the torporarousal cycle. Thus, hibernators may pre-emptively increase antioxidant defense in preparation for hibernation in order to manage the prolonged and repeated torpor-arousal cycle and muscle atrophy induced by disuse, i.e., "Preparation for Oxidative Stress" (Giraud-Billoud et al., 2019), especially in the SOL and EDL. In the GAS, however, the Nrf2 signaling pathway was only activated during the PRE, IBA, and ET states, but not during the LT and POST states. In the GAS, Nrf2 increased significantly during the PRE period, but the level of downstream antioxidant enzymes did not change significantly. We speculated that this may be because the sensitivity of antioxidant enzymes in the GAS is low, the reaction is slow, and the changes in antioxidant enzymes have not yet appeared. Conversely, because of the low sensitivity of antioxidant enzymes in the GAS, the antioxidant defense signaling pathway needs to be initiated earlier. In conclusion, activation of the Nrf2/Keap1 antioxidant pathway up-regulated antioxidant enzymes to eliminate excess ROS, which may play a vital role in skeletal muscle antioxidant defense. Furthermore, different antioxidant patterns were observed among the different types of skeletal muscle in the Daurian ground squirrels during hibernation.

\section{CONCLUSION}

Our study demonstrated that hibernating ground squirrels cope with multiple oxidative stress conditions during hibernation via up-regulation of the protein and activity levels of multiple antioxidant enzymes, thus resulting in lower ROS levels (lower $\mathrm{H}_{2} \mathrm{O}_{2}$ and MDA content) and prevention of oxidative damage. The elevated antioxidant defense capacity mediated by the Nrf2/Keap1 signaling pathway may be an important mechanism of resistance to ischemia-reperfusion and disuseinduced oxidative damage over the torpor-arousal cycle. In addition, IBA is an important period for hibernating animals to down-regulate ROS and prevent hibernationinduced oxidative stress. Under conditions of physiological suppression during hibernation, the expression levels of various antioxidant proteins were up-regulated, further suggesting that enhancement of antioxidant defense ability is crucial for coping with high stress environments and achieving successful hibernation. Furthermore, different antioxidant patterns and oxidative stress levels were observed among the different types of skeletal muscle in the ground squirrels during hibernation. The current study not only provides a new 
perspective for understanding the adaptation mechanisms of hibernating squirrels to distinct ecological environments, but also provides a theoretical basis for novel therapies to treat ischemiareperfusion damage-related diseases. Furthermore, this research could also be used to better understand how to protect organs from repeated ischemia-reperfusion.

\section{DATA AVAILABILITY STATEMENT}

All datasets generated for this study are included in the article/Supplementary Material.

\section{ETHICS STATEMENT}

The animal study was reviewed and approved by the Laboratory Animal Care Committee of the China Ministry of Health.

\section{REFERENCES}

Aldridge, G. M., Podrebarac, D. M., Greenough, W. T., and Weiler, I. J. (2008). The use of total protein stains as loading controls: an alternative to high-abundance single-protein controls in semi-quantitative immunoblotting. J. Neurosci. Methods 172, 250-254. doi: 10.1016/j.jneumeth.2008.05.003

Allan, M. E., and Storey, K. B. (2012). Expression of NF-kappaB and downstream antioxidant genes in skeletal muscle of hibernating ground squirrels, Spermophilus tridecemlineatus. Cell Biochem. Funct. 30, 166-174. doi: 10.1002/ cbf. 1832

Baird, L., and Dinkova-Kostova, A. T. (2011). The cytoprotective role of the Keap1-Nrf2 pathway. Arch. Toxicol. 85, 241-272. doi: 10.1007/s00204-0110674-5

Barja de Quiroga, G. (1992). Brown fat thermogenesis and exercise: two examples of physiological oxidative stress? Free Radic. Biol. Med. 13, 325-340. doi: 10.1016/0891-5849(92)90180-o

Bell, R. A., Smith, J. C., and Storey, K. B. (2014). Purification and properties of glyceraldehyde-3-phosphate dehydrogenase from the skeletal muscle of the hibernating ground squirrel, Ictidomys tridecemlineatus. PeerJ. 2:e634. doi: $10.7717 /$ peerj.634

Blaisdell, F. W. (2002). The pathophysiology of skeletal muscle ischemia and the reperfusion syndrome: a review. Cardiovasc. Surg. 10, 620-630. doi: 10.1016/ s0967-2109(02)00070-4

Brown, J. C., Chung, D. J., Belgrave, K. R., and Staples, J. F. (2012). Mitochondrial metabolic suppression and reactive oxygen species production in liver and skeletal muscle of hibernating thirteen-lined ground squirrels. Am. J. Physiol. Regul. Integr. Comp. Physiol. 302, R15-R28. doi: 10.1152/ajpregu.00230.2011

Bryan, H. K., Olayanju, A., Goldring, C. E., and Park, B. K. (2013). The Nrf2 cell defence pathway: keapl-dependent and -independent mechanisms of regulation. Biochem. Pharmacol. 85, 705-717. doi: 10.1016/j.bcp.2012.11.016

Carden, D. L., and Granger, D. N. (2000). Pathophysiology of ischaemiareperfusion injury. J. Pathol. 190, 255-266. doi: 10.1002/(sici)10969896(200002)190:3<255::aid-path526>3.0.co;2-6

Carey, H. V., Andrews, M. T., and Martin, S. L. (2003). Mammalian hibernation: cellular and molecular responses to depressed metabolism and low temperature. Physiol. Rev. 83, 1153-1181. doi: 10.1152/physrev.00008.2003

Chainy, G. B., Paital, B., and Dandapat, J. (2016). An overview of seasonal changes in oxidative stress and antioxidant defence parameters in some invertebrate and vertebrate species. Scientifica 2016:6126570. doi: 10.1155/2016/6126570

Chanas, S. A., Jiang, Q., McMahon, M., McWalter, G. K., McLellan, L. I., Elcombe, C. R., et al. (2002). Loss of the Nrf2 transcription factor causes a marked reduction in constitutive and inducible expression of the glutathione S-transferase Gsta1, Gsta2, Gstm1, Gstm2, Gstm3 and Gstm4 genes in the livers of male and female mice. Biochem. J. 365, 405-416. doi: 10.1042/BJ20020320

\section{AUTHOR CONTRIBUTIONS}

YW, JZ, and YG conceived and designed the experiments. YW, $\mathrm{XY}$, and XP performed the experiments. YW, HW, SX, and HC analyzed the data. YW and YG wrote and revised the manuscript.

\section{FUNDING}

This work was supported by funds from the National Natural Science Foundation of China (No. 31772459).

\section{SUPPLEMENTARY MATERIAL}

The Supplementary Material for this article can be found online at: https://www.frontiersin.org/articles/10.3389/fphys. 2019.01449/full\#supplementary-material

Chang, H., Jiang, S. F., Dang, K., Wang, H. P., Xu, S. H., and Gao, Y. F. (2016). iTRAQ-based proteomic analysis of myofibrillar contents and relevant synthesis and proteolytic proteins in soleus muscle of hibernating Daurian ground squirrels (Spermophilus dauricus). Proteome Sci. 14:16. doi: 10.1186/ s12953-016-0105-x

Cheng, G., Zielonka, M., Dranka, B., Kumar, S. N., Myers, C. R., Bennett, B., et al. (2018). Detection of mitochondria-generated reactive oxygen species in cells using multiple probes and methods: potentials, pitfalls, and the future. J. Biol. Chem. 293, 10363-10380. doi: 10.1074/jbc.RA118.003044

Circu, M. L., and Aw, T. Y. (2010). Reactive oxygen species, cellular redox systems, and apoptosis. Free Radic. Biol. Med. 48, 749-762. doi: 10.1016/j.freeradbiomed. 2009.12.022

Dark, J., and Miller, D. R. (1997). Metabolic fuel privation in hibernating and awake ground squirrels. Physiol. Behav. 63, 59-65. doi: 10.1016/s0031-9384(97) 00389-2

Dittmer, A., and Dittmer, J. (2006). Beta-actin is not a reliable loading control in western blot analysis. Electrophoresis 27, 2844-2845. doi: 10.1002/elps. 200500785

Eddy, S. F., McNally, J. D., and Storey, K. B. (2005). Up-regulation of a thioredoxin peroxidase-like protein, proliferation-associated gene, in hibernating bats. Arch. Biochem. Biophys. 435, 103-111. doi: 10.1016/j.abb.2004.11.020

Espinosa-Diez, C., Miguel, V., Mennerich, D., Kietzmann, T., Sanchez-Perez, P., Cadenas, S., et al. (2015). Antioxidant responses and cellular adjustments to oxidative stress. Redox Biol. 6, 183-197. doi: 10.1016/j.redox.2015.07.008

Ferguson, R. E., Carroll, H. P., Harris, A., Maher, E. R., Selby, P. J., and Banks, R. E. (2005). Housekeeping proteins: a preliminary study illustrating some limitations as useful references in protein expression studies. Proteomics 5, 566-571. doi: 10.1002/pmic.200400941

Frank, C. L. (1992). The influence of dietary fatty acids on hibernation by goldenmantled ground squirrels (Spermophilus lateralis). Physiol. Zool. 65, 906-920. doi: 10.1086/physzool.65.5.30158549

Fu, W., Hu, H., Dang, K., Chang, H., Du, B., Wu, X., et al. (2016). Remarkable preservation of $\mathrm{Ca}(2+)$ homeostasis and inhibition of apoptosis contribute to anti-muscle atrophy effect in hibernating Daurian ground squirrels. Sci. Rep. 6:27020. doi: 10.1038/srep27020

Gao, Y. F., Wang, J., Wang, H. P., Feng, B., Dang, K., Wang, Q., et al. (2012). Skeletal muscle is protected from disuse in hibernating dauria ground squirrels. Comp. Biochem. Physiol. A Mol. Integr. Physiol. 161, 296-300. doi: 10.1016/j. cbpa.2011.11.009

Geiser, F. (2004). Metabolic rate and body temperature reduction during hibernation and daily torpor. Annu. Rev. Physiol. 66, 239-274. doi: 10.1146/ annurev.physiol.66.032102.115105

Geiser, F. (2013). Hibernation. Curr. Biol. 23, R188-R193. doi: 10.1016/j.cub.2013. 01.062 
Giraud-Billoud, M., Rivera-Ingraham, G. A., Moreira, D. C., Burmester, T., CastroVazquez, A., Carvajalino-Fernandez, J. M., et al. (2019). Twenty years of the 'preparation for oxidative stress' (POS) theory: ecophysiological advantages and molecular strategies. Comp. Biochem. Physiol. A Mol. Integr. Physiol. 234, 36-49. doi: 10.1016/j.cbpa.2019.04.004

Gunstone, F. D. (1996). Fatty Acid and Lipid Chemistry. Maryland: Aspen Publishers.

Halliwell, B. C., and Cross, C. E. (1994). Oxygen-derived species: their relation to human disease and environmental stress. Environ. Health Perspect. 102, 5-12. doi: 10.1289/ehp.94102s105

Hermes-Lima, M., and Zenteno-Savin, T. (2002). Animal response to drastic changes in oxygen availability and physiological oxidative stress. Comp. Biochem. Physiol. C Toxicol. Pharmacol. 133, 537-556. doi: 10.1016/s15320456(02)00080-7

James, R. S., Staples, J. F., Brown, J. C., Tessier, S. N., and Storey, K. B. (2013). The effects of hibernation on the contractile and biochemical properties of skeletal muscles in the thirteen-lined ground squirrel, Ictidomys tridecemlineatus. J. Exp. Biol. 216, 2587-2594. doi: 10.1242/jeb.080663

Kalyanaraman, B. (2013). Teaching the basics of redox biology to medical and graduate students: oxidants, antioxidants and disease mechanisms. Redox Biol. 1, 244-257. doi: 10.1016/j.redox.2013.01.014

Kalyanaraman, B., Darley-Usmar, V., Davies, K. J., Dennery, P. A., Forman, H. J., Grisham, M. B., et al. (2012). Measuring reactive oxygen and nitrogen species with fluorescent probes: challenges and limitations. Free Radic. Biol. Med. 52, 1-6. doi: 10.1016/j.freeradbiomed.2011.09.030

Kim, Y. C., Masutani, H., Yamaguchi, Y., Itoh, K., Yamamoto, M., and Yodoi, J. (2001). Hemin-induced activation of the thioredoxin gene by Nrf2. A differential regulation of the antioxidant responsive element by a switch of its binding factors. J. Biol. Chem. 276, 18399-18406. doi: 10.1074/jbc.M10010 3200

Kohen, R., and Nyska, A. (2002). Oxidation of biological systems: oxidative stress phenomena, antioxidants, redox reactions, and methods for their quantification. Toxicol. Pathol. 30, 620-650. doi: 10.1080/01926230290166724

Ladner, C. L., Yang, J., Turner, R. J., and Edwards, R. A. (2004). Visible fluorescent detection of proteins in polyacrylamide gels without staining. Anal. Biochem. 326, 13-20. doi: 10.1016/j.ab.2003.10.047

Li, M., and Zhang, A. (2015). Mechanism and prevention of skeletal muscle ischemia-reperfusion injury. Chin. J. Med. Res. 44, 183-185.

Li, R., and Shen, Y. (2013). An old method facing a new challenge: re-visiting housekeeping proteins as internal reference control for neuroscience research. Life Sci. 92, 747-751. doi: 10.1016/j.lfs.2013.02.014

Li, R. H., Li, J., Kan, S. L., and Zhang, X. N. (2017). The protective effects of fasciotomy on reperfusion injury of skeletal muscle of rabbits. Biomed. Res. Int. 2017:7238960. doi: 10.1155/2017/7238960

Lin, H. P., Zheng, Y. Q., Zhou, Z. P., Wang, G. X., and Guo, P. F. (2018). Ryanodine receptor antagonism alleviates skeletal muscle ischemia reperfusion injury by modulating TNF-alpha and IL-10. Clin. Hemorheol. Microcirc. 70, 51-58. doi: 10.3233/CH- 170276

Liu, Y., Zhang, H., Mou, Y., and He, C. (2007). Mechanism of skeletal muscle ischemia/reperfusion injury. Chin. J. Clin. Rehabil. Tissue Eng. Res. 11, 9186-9189.

Lushchak, V. I. (2011). Adaptive response to oxidative stress: bacteria, fungi, plants and animals. Comp. Biochem. Physiol. C Toxicol. Pharmacol. 153, 175-190. doi: 10.1016/j.cbpc.2010.10.004

Lushchak, V. I. (2012). Glutathione homeostasis and functions: potential targets for medical interventions. J. Amino Acids 2012:736837. doi: 10.1155/2012/736837

Lushchak, V. I. (2014). Free radicals, reactive oxygen species, oxidative stress and its classification. Chem. Biol. Interact. 224, 164-175. doi: 10.1016/j.cbi.2014.10.016

Ma, Y. L., Zhu, X., Rivera, P. M., Toien, O., Barnes, B. M., LaManna, J. C., et al. (2005). Absence of cellular stress in brain after hypoxia induced by arousal from hibernation in Arctic ground squirrels. Am. J. Physiol. Regul. Integr. Comp. Physiol. 289, R1297-R1306. doi: 10.1152/ajpregu.00260.2005

Mathers, K. E., McFarlane, S. V., Zhao, L., and Staples, J. F. (2017). Regulation of mitochondrial metabolism during hibernation by reversible suppression of electron transport system enzymes. J. Comp. Physiol. B 187, 227-234. doi: 10.1007/s00360-016-1022-0

Morin, P. Jr., Ni, Z., McMullen, D. C., and Storey, K. B. (2008). Expression of $\mathrm{Nrf} 2$ and its downstream gene targets in hibernating 13-lined ground squirrels,
Spermophilus tridecemlineatus. Mol. Cell Biochem. 312, 121-129. doi: 10.1007/ s11010-008-9727-3

Morin, P. Jr., and Storey, K. B. (2007). Antioxidant defense in hibernation: cloning and expression of peroxiredoxins from hibernating ground squirrels, Spermophilus tridecemlineatus. Arch. Biochem. Biophys. 461, 59-65. doi: 10. 1016/j.abb.2007.01.035

Motohashi, H., and Yamamoto, M. (2004). Nrf2-Keap1 defines a physiologically important stress response mechanism. Trends Mol. Med. 10, 549-557. doi: 10.1016/j.molmed.2004.09.003

Muleme, H. M., Walpole, A. C., and Staples, J. F. (2006). Mitochondrial metabolism in hibernation: metabolic suppression, temperature effects, and substrate preferences. Physiol. Biochem. Zool. 79, 474-483. doi: 10.1086/501053

$\mathrm{Ni}$, Z., and Storey, K. B. (2010). Heme oxygenase expression and Nrf2 signaling during hibernation in ground squirrels. Can. J. Physiol. Pharmacol. 88, 379-387. doi: 10.1139/Y10-017

Ohta, H., Okamoto, I., Hanaya, T., Arai, S., Ohta, T., and Fukuda, S. (2006). Enhanced antioxidant defense due to extracellular catalase activity in Syrian hamster during arousal from hibernation. Comp. Biochem. Physiol. C Toxicol. Pharmacol. 143, 484-491. doi: 10.1016/j.cbpc.2006.05.002

Okamoto, I., Kayano, T., Hanaya, T., Arai, S., Ikeda, M., and Kurimoto, M. (2006). Up-regulation of an extracellular superoxide dismutase-like activity in hibernating hamsters subjected to oxidative stress in mid- to late arousal from torpor. Comp. Biochem. Physiol. C Toxicol. Pharmacol. 144, 47-56. doi: 10.1016/j.cbpc.2006.05.003

Park, E. Y., and Rho, H. M. (2002). The transcriptional activation of the human copper/zinc superoxide dismutase gene by 2,3,7,8-tetrachlorodibenzo-p-dioxin through two different regulator sites, the antioxidant responsive element and xenobiotic responsive element. Mol. Cell Biochem. 240, 47-55.

Peter, J. B., Barnard, R. J., Edgerton, V. R., Gillespie, C. A., and Stempel, K. E. (1972). Metabolic profiles of three fiber types of skeletal muscle in guinea pigs and rabbits. Biochemistry 11, 2627-2633. doi: 10.1021/bi00764a013

Rouble, A. N., Tessier, S. N., and Storey, K. B. (2014). Characterization of adipocyte stress response pathways during hibernation in thirteen-lined ground squirrels. Mol. Cell Biochem. 393, 271-282. doi: 10.1007/s11010-014-2070-y

Ruuge, E. K., Ledenev, A. N., Lakomkin, V. L., Konstantinov, A. A., and Ksenzenko, M. (1991). Free radical metabolites in myocardium during ischemia and reperfusion. Am. J. Physiol. 261, 81-86. doi: 10.1152/ajplung.1991.261. 4.L81

Sanderson, T. H., Reynolds, C. A., Kumar, R., Przyklenk, K., and Huttemann, M. (2013). Molecular mechanisms of ischemia-reperfusion injury in brain: pivotal role of the mitochondrial membrane potential in reactive oxygen species generation. Mol. Neurobiol. 47, 9-23. doi: 10.1007/s12035-012-8344-z

Soukri, A., Valverde, F., Hafid, N., Elkebbaj, M. S., and Serrano, A. (1996). Occurrence of a differential expression of the glyceraldehyde-3-phosphate dehydrogenase gene in muscle and liver from euthermic and induced hibernating jerboa (Jaculus orientalis). Gene 181, 139-145. doi: 10.1016/s03781119(96)00494-5

Storey, K. B. (2010). Out cold: biochemical regulation of mammalian hibernation a mini-review. Gerontology 56, 220-230. doi: 10.1159/000228829

Sun, Z., Zhang, S., Chan, J. Y., and Zhang, D. D. (2007). Keap1 controls postinduction repression of the Nrf2-mediated antioxidant response by escorting nuclear export of Nrf2. Mol. Cell Biol. 27, 6334-6349. doi: 10.1128/ MCB.00630-07

Toien, O., Drew, K. L., Chao, M. L., and Rice, M. E. (2001). Ascorbate dynamics and oxygen consumption during arousal from hibernation in Arctic ground squirrels. Am. J. Physiol. Regul. Integr. Comp. Physiol. 281, R572-R583.

Turrens, J. F. (2003). Mitochondrial formation of reactive oxygen species. J. Physiol. 552, 335-344. doi: 10.1113/jphysiol.2003.049478

Valko, M., Leibfritz, D., Moncol, J., Cronin, M. T., Mazur, M., and Telser, J. (2007). Free radicals and antioxidants in normal physiological functions and human disease. Int. J. Biochem. Cell Biol. 39, 44-84. doi: 10.1016/j.biocel.2006. 07.001

Vigelso, A., Dybboe, R., Hansen, C. N., Dela, F., Helge, J. W., and Guadalupe Grau, A. (2015). GAPDH and beta-actin protein decreases with aging, making stain-Free technology a superior loading control in Western blotting of human skeletal muscle. J. Appl. Physiol. 118, 386-394. doi: 10.1152/japplphysiol.00840. 2014 
Vucetic, M., Stancic, A., Otasevic, V., Jankovic, A., Korac, A., Markelic, M., et al. (2013). The impact of cold acclimation and hibernation on antioxidant defenses in the ground squirrel (Spermophilus citellus): an update. Free Radic. Biol. Med. 65, 916-924. doi: 10.1016/j.freeradbiomed.2013.08.188

Wang, J. Z., and Yin, L. H. (2013). Pathophysiology. Beijing: People's medical publishing house.

Wei, Y., Gong, L., Fu, W., Xu, S., Wang, Z., Zhang, J., et al. (2018a). Unexpected regulation pattern of the IKKbeta/NF-kappaB/MuRF1 pathway with remarkable muscle plasticity in the Daurian ground squirrel (Spermophilus dauricus). J. Cell Physiol. 233, 8711-8722. doi: 10.1002/jcp.26751

Wei, Y., Zhang, J., Xu, S., Peng, X., Yan, X., Li, X., et al. (2018b). Controllable oxidative stress and tissue specificity in major tissues during the torpor-arousal cycle in hibernating Daurian ground squirrels. Open. Biol. 8:180068. doi: 10. 1098/rsob.180068

Winterbourn, C. C. (2008). Reconciling the chemistry and biology of reactive oxygen species. Nat. Chem. Biol. 4, 278-286. doi: 10.1038/nchembio.85

Wu, C. W., and Storey, K. B. (2014). FoxO3a-mediated activation of stress responsive genes during early torpor in a mammalian hibernator. Mol. Cell Biochem. 390, 185-195. doi: 10.1007/s11010-014-1969-7

Xu, R., Andres-Mateos, E., Mejias, R., MacDonald, E. M., Leinwand, L. A., Merriman, D. K., et al. (2013). Hibernating squirrel muscle activates the endurance exercise pathway despite prolonged immobilization. Exp. Neurol. 247, 392-401. doi: 10.1016/j.expneurol.2013.01.005

Yang, C. X., He, Y., Gao, Y. F., Wang, H. P., and Goswami, N. (2014). Changes in calpains and calpastatin in the soleus muscle of Daurian ground squirrels during hibernation. Comp. Biochem. Physiol. A Mol. Integr. Physiol. 176, 26-31. doi: 10.1016/j.cbpa.2014.05.022
Yin, Q., Ge, H., Liao, C. C., Liu, D., Zhang, S., and Pan, Y. H. (2016). Antioxidant Defenses in the brains of bats during hibernation. PLoS One 11:e0152135. doi: 10.1371/journal.pone.0152135

Zhang, J., Wei, Y., Qu, T., Wang, Z., Xu, S., Peng, X., et al. (2019). Prosurvival roles mediated by the PERK signaling pathway effectively prevent excessive endoplasmic reticulum stress-induced skeletal muscle loss during high-stress conditions of hibernation. J. Cell Physiol. 234, 19728-19739. doi: 10.1002/jcp. 28572

Zhang, Y., Li, C., Zhang, H., Li, W., Li, J., and Wang, X. (2018). Color-coded digital subtraction angiography for assessing acute skeletal muscle ischemiareperfusion injury in a rabbit model. Acad. Radiol. 25, 1609-1616. doi: 10.1016/ j.acra.2018.03.020

Zong, H., Li, X., Lin, H., Hou, C., and Ma, F. (2017). Lipoxin A4 pretreatment mitigates skeletal muscle ischemia-reperfusion injury in rats. Am. J. Transl. Res. $9,1139-1150$

Conflict of Interest: The authors declare that the research was conducted in the absence of any commercial or financial relationships that could be construed as a potential conflict of interest.

Copyright (c) 2019 Wei, Zhang, Yan, Peng, Xu, Chang, Wang and Gao. This is an open-access article distributed under the terms of the Creative Commons Attribution License (CC BY). The use, distribution or reproduction in other forums is permitted, provided the original author(s) and the copyright owner(s) are credited and that the original publication in this journal is cited, in accordance with accepted academic practice. No use, distribution or reproduction is permitted which does not comply with these terms. 